\title{
Mixed-Integer Programming Based Techniques for Resource Allocation in Underlay Cognitive Radio Networks: A Survey
}

\author{
Attahiru S. Alfa, B. T. Maharaj, Shruti Lall, Sougata Pal
}

\begin{abstract}
For about the past decade and a half research efforts into cognitive radio networks (CRNs) have increased dramatically. This is because CRN is recognized as a technology that has the potential to squeeze the most out of the existing spectrum and hence virtually increase the effective capacity of a wireless communication system. The resulting increased capacity is still a limited resource and its optimal allocation is a critical requirement in order to realize its full benefits. Allocating these additional resources to the secondary users (SUs) in a CRN is an extremely challenging task and integer programming based optimization tools have to be employed to achieve the goals which include, among several aspects, increasing SUs throughput without interfering with the activities of primary users (PUs). The theory of the optimization tools that can be used for resource allocations (RA) in CRN have been well established in the literature; convex programming is one of them, in fact the major one. However when it comes to application and implementation, it is noticed that the practical problems do not fit exactly into the format of well established tools and researchers have to apply approximations of different forms to assist in the process. In this survey paper, the optimization tools that have been applied to RA in CRNs are reviewed. In some instances the limitations of techniques used are pointed out and creative tools developed by researchers to solve the problems are identified. Some ideas of tools to be considered by researchers are suggested, and direction for future research in this area in order to improve on the existing tools are presented.
\end{abstract}

Index Terms: Cognitive radio networks, Resource allocation, Optimization, Mixed-integer programming.

\section{INTRODUCTION}

Cognitive radio (CR) [1],[2],[3], is a wireless communication technology which is evolving in a rapid pace. One of the main objectives of CR networks (CRN) is the efficient utilization of the radio spectrum. It capitalizes on making use of the under-utilized radio frequency spectrum by letting unlicensed secondary users (SUs) co-exist with the licensed primary users (PUs) of a primary radio network without the SUs interfering with the activities of the PUs.

There are two key components, among others, that are needed for the success of CRN. The first is proper sensing to detect under-utilized spectrum and the other is how to efficiently al-

Attahiru S. Alfa is with Department of Electrical and Computer Engineering, University of Manitoba, Winnipeg, MB, Canada R3T 5V6 (e-mail: Attahiru.Alfa@umanitoba.ca) and Department of Electrical, Electronic and Computer Engineering, University of Pretoria, Pretoria, South Africa, 0002

B.T.Maharaj, S. Lall and S. Pal are with Department of Electrical, Electronic and Computer Engineering, University of Pretoria, Pretoria, South Africa, 0002 (e-mail: Sunil.Maharaj@up.ac.za). locate this spectrum hole to the SUs. Several research activities are on-going in the area of sensing. Most of the works are based on statistical analysis of sensing on how to make inferences on signals detected, and the other is on finding optimal sensing strategies. Our interest is not on those aspects. Our interest is mainly on the tools used to allocate, the SUs, the sensed to be idle spectrum which we refer to as a resource. Resource allocation (RA) problems in a CR environment are currently being extensively investigated by researchers in the telecommunications industry. Specifically how to allocate resources in an efficient manner to SUs in a CRN is the key issue. RA is carried out by using optimization tools, and the most common ones are based on some form of integer programming.

Let us briefly explain what an RA problem in CRN is about. Generally we have a PU who is licensed to use a particular channel. SUs are allowed to access this channel if they can do so without interfering with the PU. This process may be achieved in two different manners. One approach is for the SU to find when the PU channel is idle and access it, keeping in mind that it will have to give it up as soon as the PU comes back to the channel. This scenario is termed overlay. The other approach is for the SU to access the channel even when it is occupied by the PU, but the SU does so at a considerably low enough power that will not create interference to the PU. This second approach is termed underlay. Our interest is in the underlay scenario. In this underlay scenario which SU is allowed to access which channel and at what power they can transmit has to be managed appropriately in order for the available limited resources to be optimally well managed. This is what is generally termed resource allocation (RA) in CRN. Mathematical programming tools have been employed by telecommunication researchers in the attempt to find how to allocate the resources optimally.

Integer programming (IP) is a mathematical tool commonly used to select integer variables that can help determine an optimal solution to a problem. For example, given several PU channels for which SUs can transmit at a low enough power without interfering with the PU, deciding which SU to assign each channel and how many channels to assign in order to maximize some major objective of the system can be seen as an integer programming problem. If in addition a decision has to be made on power allocation also then we end up with a mixed-integer problem. Optimization of RA problems in a CRN have generally been carried out by IP or a different member from its family. IP family includes integer linear programming (ILP), integer non linear programming (INLP), mixed integer linear programming (MILP), mixed integer non linear programming (MINLP), binary integer linear programming (BILP) etc. Mixed-integer 
programming (MIP) includes both MILP and MINLP. Finding an optimal solution of an IP problem is extremely computationally complex because they are generally Non-deterministic Polynomial-time hard (NP-hard) [4]. Due to this complexity, IP problems generally have a sub-optimal solution to the original problems. This paper contains a literature review from 2006 onwards regarding the applications of IP for finding a suitable sub-optimal solution for different RA problems in CRNs. We selected the year 2006 because from our observations most of the publication activities for this subject got into full swing after 2005 , and before then the activities were limited. In that case we hope that papers before 2006 have been cited by papers that were published after that year. As expected, it is not unusual to inadvertently omit some references, especially when there have been lots of published papers in the area in a short period of time (2006 to 2015). For this we apologize in advance, as this was not intentional.

The remainder of this paper is organized as follows: Section II presents the basic summary of tools used in optimization and Section III classifies the objective functions of the resource allocation problems studied. A survey of the tools commonly used in radio resource allocation for cognitive radio networks is presented in Section IV. Challenges and open problems are presented in Section V and finally in Section VI conclusions are presented.

\section{BASIC SUMMARY OF OPTIMIZATION TOOLS}

In this section we present a quick overview of basic optimization techniques that mixed-integer programming is based on. This is to help us set the stage for introducing some terminologies for our problem before we present a small example of how it is applied to a CRN resource allocation problem. Consider an $n$ column vector $\mathbf{x} \in \Re$ and an $m$ column vector $\mathbf{y} \in \mathcal{Z}=$ $\{0,1,2,3, \cdots\}$. Suppose we want to find the values of these two vectors for which a function $f(\mathbf{x}, \mathbf{y})$ is minimum, given that there are a set of constraints $g_{i}(\mathbf{x}, \mathbf{y}) \leq b_{i}, i=1,2, \cdots, r$, and that each variable is non-negative. Here $f:\left(\Re^{n}, \mathcal{Z}^{m}\right) \rightarrow \Re$, and $g_{i}:\left(\Re^{n}, \mathcal{Z}^{m}\right) \rightarrow \Re, \forall i$. This problem can be written as

$$
\begin{gathered}
\min _{\mathbf{x}, \mathbf{y}} \quad z=f(\mathbf{x}, \mathbf{y}) \\
\text { s.t. } g_{i}(\mathbf{x}, \mathbf{y}) \leq b_{i}, i=1,2, \cdots, r, \\
x_{k} \geq 0, \quad k=1,2, \cdots, n, \\
y_{j} \in \mathcal{Z}, \quad j=1,2, \cdots, m .
\end{gathered}
$$

For simplicity and later use we can also write Eq. (2) as

$$
\mathbf{g}(\mathbf{x}, \mathbf{y}) \leq \mathbf{b}
$$

where

$$
\mathbf{g}(\mathbf{x}, \mathbf{y})=\left[\begin{array}{c}
g_{1}(\mathbf{x}, \mathbf{y}) \\
g_{2}(\mathbf{x}, \mathbf{y}) \\
\vdots \\
g_{r}(\mathbf{x}, \mathbf{y})
\end{array}\right]
$$

and $\mathbf{b}=\left[b_{1}, b_{2}, \cdots, b_{r}\right]^{T}$ and for a matrix $\mathbf{A}$ we write its transpose as $\mathbf{A}^{T}$. If the problem was to maximize a function $z=$ $h(\mathbf{x}, \mathbf{y})$, that could be transformed to a form of minimization function, by letting $f(\mathbf{x}, \mathbf{y})=-h(\mathbf{x}, \mathbf{y})$, i.e. now writing

$$
\min _{\mathbf{x}, \mathbf{y}} w=f(\mathbf{x}, \mathbf{y}) .
$$

This is a mixed-integer programming problem, where the elements of the vector $\mathbf{x}$ are nonnegative and those of $\mathbf{y}$ nonnegative integer values. Eq. (1) and the lefthand side of the inequality given in Eq. (2) may be linear or non-linear. If any of them is non-linear then we have a non-linear mixed-integer programming problem.

Most of the RA problems encountered in CRN are of the general form Eq. (1) to Eq. (4), or Eq. (1) replaced by Eq. (5). The first equation is called the objective function, the remaining ones are the constraints and the variables $x_{k}$ and $y_{j}$ are the decision variables. First let us discuss the most commonly occurring types of objective functions, constraints and the decision variables in CRN. Even though several kinds of these functions and parameters are encountered in RA problems, we focus on the most occurring ones that set the stage for the complexities associated with the optimization problems for RA.

There are several types of objective functions occurring in RA for CRN of which three appear to be very prominent. Most of the other types are related to those three. The three are:

- maximization of network sum throughput,

- maximization of overall user data rate,

- maximization of user capacity.

The other types of objective functions that have been considered in the literature include; maximization of spectrum utilizations, maximization of total channel utility, maximization of fairness scaling factor, maximization of downlink channel capacity, minimization of total transmission power, and minimization of total power consumption. Even the three mentioned earlier seem to have been used interchangeably by authors. For example, the "user data rate" was used by [5] and "throughput" by [6], and "sum capacity" by [7], but essentially the functions are about the same. Even though we could have chosen to stay with the use of the terms and terminologies as used by authors in order to make it easier for readers, that may make the presentation repetitious in many instances. So we are combining all those three objective functions together into a class of "capacity" measure.

As for constraints, there are three that are very commonly occurring and they are:

- power constraint,

- constraint on interference,

- minimum data rate for users.

The most commonly occurring decision variables are:

- transmission power,

- sub-channel allocation, which results in a zero-one decision variable.

As an example of a typical optimization problem for CRN, the vector $\mathrm{x}$ could be a set of transmission power for some users that we have to decide on and the vector $\mathbf{y}$ could be subchannel allocation zero-one variables. The constraint in Eq. (2) could be interference limit constraint or power constraint, and finally Eq. (1) could be maximization of the network sum 
throughput. Generally this is not a convex optimization problem, simply because of the mixed-integer aspects. However if $y_{j}, j=1,2, \cdots, m$ are relaxed such that $y_{j} \in \Re, \forall j$, and $f(\mathbf{x}, \mathbf{y})$ and $g_{i}(\mathbf{x}, \mathbf{y}), i=1,2, \cdots, r$ are convex, then this problem is called a convex optimization and there is a considerable literature on this subject with well documented techniques for analyzing them (see [8]). An example of a method is one that is based on applying the Lagrangian duality and the Karush-KuhnTucker (KKT) conditions. The relaxation of $y_{j}$ completely removes the integer requirement for that variable which is one of the major aspects that make it non-convex, keeping in mind that there are other aspects that may render it non-convex. Hence one cannot apply the standard convex optimization techniques to the general RA problems for CRN.

Suppose $f(\mathbf{x}, \mathbf{y})$ and $g_{i}(\mathbf{x}, \mathbf{y}), i=1,2, \cdots, r$ are linear, then this problem is a classical mixed-integer linear programming problem for which one may apply the Branch and Bound method or the Lagrangian relaxation approach [9], [10]. Better still is if the variables $y$ do not have to be integers, then one may apply standard Linear Programming (LP) tools.

Essentially we have one objective function (Eq. (1)), $r$ resource constraints (Eq. (2)), $n$ non-negative variables and $m$ non-negative integer variables. Usually the $n$ non-negative variables should not pose major problems. However, satisfying the integer variable restrictions is one of the issues that we face in these optimization problems. Next is the set of $r$ resource constraints. All the constraints, $g_{i}(.,),. i=1,2, \cdots, r$, need to be convex and if we plan to use LP approach all have to be linear. Finally the objective function needs to be convex or linear if we plan to use the LP approach. It becomes clear that there are several combinations of the form of all these constraints and the objective function that can make the problem complex. When most of the constraints are linear and/or the objective function is linear there is the tendency to assess whether a linearization of the rest would lead to an approximation so that one can apply the classical LP approach, as an example. There are several other types of approximations that can be considered and will be discussed in this survey. First let us discuss the three main components of the optimization problems as encountered for CRN. We present the objective functions, the constraints and decision variables that are commonly seen in RA for CRN.

\section{AN EXAMPLE OF A GENERIC SIMPLE RA PROBLEM}

In what follows we introduce a small generic resource allocation problem and use that as the basis of our discussions in comparing the papers in the literature. Consider a simple CRN problem in which we have $K$ PU channels. There are $M$ SUs looking for access to the PU channels. Each SU, $s=1,2, \cdots, M$ has a maximum power source of $P_{\max }^{s}$. If SU $s$ is allowed to transmit on channel $k$ with power level $P_{k}^{s}$, then its capacity, $c_{k}^{s}$ will be given as $c_{k}^{s}=\log \left(1+\gamma_{k}^{s} P_{k}^{s}\right)$, where $\gamma_{k}^{s}$ is the channel to noise level ratio associated with SU $s$ transmitting on channel $k$ This is essentially a simplified Shannon's capacity formula or a formula derived from it. Whether we are dealing with capacity, throughput or data rate a version of this formula is what we use.

Let $x_{k}^{s}=1$ if channel $k$ is assigned to SU, $s$ and zero, otherwise. Generally the throughput and data rate resulting from this are directly proportional to this capacity. So in essence the total capacity assigned to this $\mathrm{SU}, s$ will be

$$
z_{s}=\sum_{k=1}^{K} c_{k}^{s} x_{k}^{s}
$$

In RA problem our interest would be to maximize the total weighted capacity for all the SUs, with the weight $w_{s}$ assigned to SU $s$. Hence the objective function of this generic problem will be

$$
\max z=\sum_{s=1}^{M} w_{s} \sum_{k=1}^{K} c_{k}^{s} x_{k}^{s} .
$$

This will be our equivalent of Eq. (1). It is a non-linear function in $P_{k}^{s}$ and in $x_{k}^{s}$.

Next we consider the constraints. The first one is that we cannot assign a channel to more than one SU. So we need the constraint

$$
\sum_{s=1}^{S} x_{k}^{s} \leq 1, \quad \forall k=1,2, \cdots, K,
$$

and also a constraint that ensures that we do not allocate more than available channels to all the SUs, i.e.

$$
\sum_{s=1}^{M} \sum_{k=1}^{K} x_{k}^{s} \leq K
$$

These two constraints will be a versions of Eq. (2) with all the variables here allowed to be only values of zero or one.

The next constraint is that we cannot allow the total power generated by an SU to exceed its power limit. So we need the constraint that

$$
\sum_{k=1}^{K} P_{k}^{s} \leq P_{\max }^{s}, \forall s=1,2, \cdots, M,
$$

which is an equivalent version of Eq. (2) with the variables allowed to assume any non-negative values.

A key requirement in $\mathrm{CRN}$ is that the $\mathrm{SU}$ should not interfere with the PU, or at least the interference should not exceed the maximum allowed level. This can be easily captured by requiring that the power reaching the PU should not exceed a particular value $P$. So the next constraint is

$$
\sum_{s=1}^{M} \sum_{k=1}^{K} P_{k}^{s} \gamma_{k}^{s} \leq P
$$

another version of Eq. (2) with the variables allowed to assume any non-negative values.

Given that SUs usually have a minimum requirement for QoS we assume that there is a constraint in this regard also. For example, an SU, $s$, may require a minimum of $\sigma_{s}$ of total number of sub-channels assigned, so this leads to the constraint

$$
\sum_{k=1}^{K} x_{k}^{s} c_{k}^{s} \geq \sigma_{s}, \forall s=1,2, \cdots, M
$$

Finally we have the two critical but common constraints, i.e. that of zero-one on $x_{k}^{s}$ and non-negativity on $P_{k}^{s}$, both written as

$$
x_{k}^{s}=\{0,1\} \text {. }
$$




$$
P_{k}^{s} \geq 0
$$

In summary, Eqs (9) to (16) form the RA problem for this simple example. As one can see, in its simplest form, the objective function in non-linear, and Constraint (14) is non-linear. Also one variable, $x_{k}^{s}$ is zero-one while $P_{k}^{s}$ is a simple non-negative variable. So unless there is a significantly different problem studied, non-linearity and integer variables (zero-one) are unavoidable in the formulations. That is why in general we have a non-linear MIP problem for RA. The issue now is how it has been handled in the literature.

An optimization problem is determined by its objective function, constraints and decision variables. The objective function is one expression whilst the constraints could involve several inequalities and equalities, and the decision variables are usually many. In order to present this survey in a very structured form we classify the literature according to the objective function types for discussion. The alternative approach is that we classify each problem by the general class of the optimization type it belongs, i.e. whether it is a convex optimization problem or not. This approach could lead to just one class, i.e. nonconvex programming problem, as most of them look that way and only very limited number fit into the convex programming class simply because they were modified. It was observed that only few papers did declare whether their problems were convex programming or not. This we can understand, as such a task is usually challenging for most practical realistic problems. In fact trying to develop simple approaches for assisting in establishing convexity of an optimization is very important and should be one of the future works in this area of research.

\section{CLASSIFICATION BY OBJECTIVE FUNCTION TYPES}

As seen in the simple generic problem formulation, provided the objective is to maximize throughput or capacity or data rate, chances are that the objective function will involve the Shannon capacity formula. Hence the objective function will be nonlinear. Of the 79 papers surveyed, 16 of them claim to have linear objective functions. Of the 16 papers, at least 10 of them had either sum data rate or throughput as their objective functions. It is therefore important for us to discuss how they were able to carve out a linear function as an objective in those situations. This should be of interest to researchers and help in understanding how to handle other problems in the future.

So, the problems are classified according to the two most commonly used types that occur; Linear or Non-linear objective functions. The most important aspect of an objective function as it relates to solving an optimization problem is whether it is linear or not. If it is a linear function, then we may start to think of an LP based method, hoping that the constraints may be linear or a good number of them are linear and that the rest if not linear can be easily approximated by linear functions. Of course, if most of the constraints are non-linear and can not be well approximated by linear functions then an LP based method may not be a good candidate approach to consider.

If the objective function is non-linear, the first thought is whether it is a convex or concave function. For a minimization problem a convex function has properties that make it amenable to convex programming. This does not imply that a concave function does not have an optimal solution. It is just that the solution, if it exists in this case, will be at an edge or corner. Another factor that we look for is whether the function can be linearized as an approximation, with the hope that most of the constraints are linear and those that are not linear can be linearized. If the objective function, $f(\mathbf{x}, \mathbf{y})$ is convex and the constraints, $\mathbf{g}(\mathbf{x}, \mathbf{y})$ are convex then one has the chance to apply standard convex programming techniques, i.e. use the Lagrangian Duality and apply the well-known Karush-Kuhn-Tucker (KKT) conditions.

\section{A. Linear Objective Function}

Of all the papers surveyed, the ones which were trying to maximize data rates and which have linear objective functions include, [11],[12], [13],[14],[15],[16]. The initial formulation in [11] was non-linear objective function and some of the constraints were non-linear. This is because the equation for the required power for a given BER is non-linear and as a result the objective function was non-linear. However, the authors were able to reformulate the problem to an integer linear programming problem by defining a binary variable $x_{k, n, c}$ which is equal to 1 if the $n^{t h}$ subcarrier is assigned to the $k^{\text {th }} \mathrm{SU}$ which transmits $c$ number of bits per symbol, and also another variable $p_{k, n, c}$ which is the transmission power required by the $k^{t h} \mathrm{SU}$ on the $n^{\text {th }}$ subcarrier to transmit $c$ number of bits per symbol. This is a major and very useful transformation. The idea was used by Awoyemi et al. [17] in solving a class of RA problems for heterogeneous CRN. Mitran et al. [12] had a pure ILP right from the start. The decision variable involves a binary term $S_{i j z}$ which equals 1 if sub-channel $j$ allocated to SU $i$ uses transmission mode $z$ which transmits at a rate $R_{z}$. Hence the objective function was of the $\max \min _{S_{i, j, z}, i} \sum_{j} \sum_{z} R_{z} S_{i j z}$, which is linear. However, two of the constraints consist of a term $f_{i j}(z)$ which is the minimum required transmit power associated with $s_{i j z}$ and it is assumed to be given. Without this been given the problem could have probably been non-linear. The linearity of the objective function was achieved by predetermining the rates associated with each transmission mode and then formulating an objective function with channel selection that maximizes total rate. Shi and Hou [13], even though had linear objective function in their problem some of the constraints were non-linear; they are the constraints that involve power allocation. The authors were able to develop a clever approximation for those nonlinear constraints and then obtained upper bounds for the solution of the problem. They pointed out that their bounds and feasible solution gap showed that they have good solutions. Shi and Hou [13] were able to achieve linearity of objective function by trying to maximize a function that is linear in the minimum rate and multiplied by a factor $K$ they call scaling factor. As a result the objective function is linear. In both their papers [14],[15] the authors considered optimal framework for the design of spectrum sharing and flow routing in CRN with interference consideration. Their problems are of the mixed-integer linear programming types. The authors however developed heuristics for solving the problem. They did not explore the structures of the problem to see whether they could exploit them. They achieved linearity of the objective functions by maximizing the percentage traffic demand as a pseudo measure of data rate. Shu 
and Krunz [16] developed a mixed-integer non-linear programming problem for joint power/rate control and channel assignment. However they were able to exploit the discrete set of rates structure and then transform the problem to a binary linear programming problem (BLP) with linear constraints. They then developed a centralized polynomial time linear programming with sequential fixing to further approximate the BLP by exploiting the structure of their problem. All these papers identified their objective functions as capturing data rates.

Another group of papers that had linear objective functions include ([18],[19],[20],[21]). The work of Rahulamathavan et al. [18] is an extension of their previous work [11] which was on OFDMA to MIMO-OFDMA. In their paper, Guo and Huang [19] considered opportunistic spectrum access in a cell covering environment. They specifically considered overlay CRN situation and studied channel assignment and power control. They achieved linearity of the objective function by using the data rate assigned to a link, which is a linear function of power, as the objective function.The resulting problem formulation was a mixed integer linear programming problem. They then used a greedy algorithm to analyze it. It is surprising that they did not report any attempts to explore any possible structure of the problem given that it is a linearly based problem. On the other hand Shih et al. [20], who obtained a linear objective function by using data rate of a route whose upper bounds are known, were able to develop a special technique for analyzing their integer linear programming problem that resulted from joint routing and spectrum allocation for multi-hop CRN. They relaxed the integer constraints and solved the resulting linear programming problem iteratively. They were very creative in their approach by fixing the values of some of the integer variables to 1 or 0 after each iteration, based on how the results turn out. They then proceed to the next iteration with some variables fixed and repeated the process until a good solution is obtained. The idea is novel and worth considering by other researchers. Wang et al. [21] ended up with a binary linear programming problem in their work on developing a spectrum sharing algorithm for throughput in CRN. Somehow they seem to assume that the transmit power is given and this allowed the problem to become linear. They first assigned power, hence avoided having to include the Shannon capacity formula in their objective function, and then carried out channel assignment. Their assumption about power allocation does not seem to be realistic. All these four papers identified their objective functions as capturing throughput.

The following papers [22],[23],[24] had objective functions that are linear and not based on capacity, throughput or data rate. Hoang and Liang [22] considered the problem of downlink channel assignment and power control for CRN, however their objective function was maximizing the total number of active subscribers in CRN. Their model was a mixed integer linear programming problem and they proposed what they called DIGA (dynamic interference graph allocation) algorithm. This work seemed like a minor extension of the authors' previous paper [23]. The linear formulation of the problem by Rahulamathavan et al. [24] is essentially similar in format to [18]. The main difference here is that the objective function involves scheduling and what they call MA (margin adaptive) problem which essentially is that of minimizing transmit power subject to data rate constraint.

An et al. [25] studied the optimization of energy efficiency in cognitive femtocell networks. The authors considered the maximization of a revenue function associated with the femtocells. The authors formulated the power control problem of standalone femtocells as a linear optimization problem and introduced a low-complexity iteration algorithm based on gradient-assisted binary search algorithm to solve it. In addition, the authors formulated the power control of collocated femtocells in a set as non-cooperative game, and used an asymptotic analysis to obtain the approximate spectrum utilization price in macrocells.

\section{B. Non-linear Objective Function}

The papers with non-linear objective functions include, [26],[5],[27],[28],[29], [30],[31],[32],[20],[33]. None of these problems were approximated by linear objective functions. Even though some papers in the literature were able to take advantage of the structure of their nonlinear problems by converting the non-linear objective functions or constraint to linear function, as pointed out in Section IVA, the papers classified as non-linear in this paragraph could not, or did not exploit any structure of their problem. This is partly because non-linearity of the objective function was not the main source of difficulties in solving the problem but rather the constraints were also very complex. In their work, Mitran et al. [26] studied a queue aware resource allocation problem for downlink system. It resulted in the formulation of a large non-linear integer programming problem. The authors then proposed heuristics based on decoupling the power allocation and rate allocation problems. On the other hand Waheed and Cai [27] used the binary particle swarm and genetic algorithm, both evolutionary methods, to solve their problem of resource allocation in CRN. Ngo et al. [28] did adopt a different approach compared to others. They used Lagrange duality idea to handle the non-convex mixednonlinear integer program arising from their RA problem. They relaxed the integrality of some of the variables and turned the problem to a convex optimization successfully. Salameh [29] on the other hand, by considering finite number of idle channels and pre-specifying maximum transmission power was able to exploit the structure of the problem and reduce it to a binary linear programming problem. Another interesting approach was adopted by Zhang and Leung [30]. They used multidimensional knapsack approach together with a greedy max-min algorithm to deal with the RA problem. Stochastic programming was used by Xie et al. [31] for the joint power allocation and beamforming - a different but related class of problem. Shin et al. [32], used geometric programming approach to deal with joint rate and power allocation problem while Li et al. [33] linearized their original formulation of the max-min rate problem which was a mixed non-linear problem. In [33] they were only able to linearize some functions, one of the constraints could not be linearized so in the end they combined what they called sequential parametric convex approximation with some heuristics to solve the problem. Generally most of the problems discussed in this paragraph did not lend themselves to simple linearization for the authors. For example, [5] has an objective function of the form 


$$
\max _{\mathbf{P}, \mathbf{a}} \sum_{m=1}^{M} w_{m} R_{m}
$$

where $w_{m}$ is the weight of the cognitive user $U_{m}$ and $R_{m}$ is the maximum transmit rate of the cognitive user $U_{m}$. Here $\mathbf{a}=\left[a_{11}, a_{12}, . ., a_{1 K}, a_{21}, \ldots a_{M K}\right]^{T}$ is the cognitive users binary channel allocation vector, where $a_{i j}$ is 1 if channel $j$ is allocated to user $i$ and zero otherwise, and $\mathbf{P}=$ $\left[P_{11}, P_{12}, . ., P_{1 K}, P_{21}, \ldots P_{M K}\right]^{T}$ is the power allocation vector, where $P_{i j}$ is the power allocated to user $i$ on channel $j$.

The variable $R_{m}$ is given as

$$
R_{m}=\sum_{k=1}^{K} a_{m k} \log _{2}\left(1+\frac{h_{m k} P_{m k}}{\Gamma \sigma_{k}^{2}}\right), \forall m .
$$

The parameters associated with this equation are defined as follows: $\Gamma$ is a constant SNR gap, $h_{m k}$ is the link gain between cognitive transmiter $U_{m}(1 \leq m \leq M)$ and its intended receiver $Y_{m}(1 \leq m \leq M)$ on subchannel $k(1 \leq k \leq K)$, and $\sigma_{k}$ is the sum of power of Gaussian noise and interference caused by primary users on subchannel $k$. Note that $K$ is the number of subchannels and $M$ is the number of secondary users.

Two commonly occurring decision variables, as earlier mentioned, are transmission power and sub-channel allocation. Transmission power is a non-negative real variable whereas subchannel allocation is a zero-one variable. Thus we find that most times we are dealing with mixed-integer programming (MIP) problems, whether linear or non-linear. So this already creates some form of difficulty.

It is obvious why this objective function was not linearized. Any attempt to carry out this linearization will lead to a very poor approximation, if not an unrealistic approximation. Suppose the $\log$ function was linearized, one still has to deal with the fact that the objective function is a sum of products, hence a nonlinear function, even though the idea of quadratic programming may then be considered. In any case, a reasonable linearization may be difficult. In addition, one of the main set of constraints is also non-linear. The constraints to the problem are given as follows

$$
\begin{aligned}
& P_{m k} \geq 0, \forall m, k, \\
& a_{m k} \in(0,1), \forall m, k, \\
& \sum_{m=1}^{M} a_{m k} \leq 1, \forall k, \\
& \frac{\sum_{k=1}^{K} \sum_{m=1}^{M} a_{m k} b_{n k} g_{m k}^{n} P_{m k}}{B_{n}} \leq T_{n}, \forall n,
\end{aligned}
$$

where the remaining parameters are known and well defined constants. The last set of constraints is interference temperature constraint and is non-linear. It is a sum of products of the transmission power and the channel allocation which is a zero-one variable. Specifically $b_{n k}$ assumes the value of 1 if sub channel $k$ is assigned to primary user $n$, or zero otherwise, $h_{m k}$ is the channel gain for CR user $m$ on link $k$ and $g_{m k}^{n}$ is its associated interference to primary user $n . B_{n}$ is the bandwidth and $T_{n}$ is the interference threshold to primary user $n$.

Thus this objective function has to be considered the way it is, i.e. as non-linear function. The question then is whether it is a well behaved function for optimization in its non-linear form. This is a maximization problem, so we want the objective function to be concave, if the variables are all real and not necessarily integers. The log component is concave, and since the variable $a_{m k}$ is a zero-one variable it follows that the objective function is concave. Hence it is a well behaved function for our purpose. We simply have to find an appropriate method for solving this problem. Most of these problems employed OFDM/OFDMA and their declared goal was to maximize data rate.

A group of papers had non-linear objective functions, in many instances in MIMO-OFDMA downlink RA, and defined their objective functions as throughput. Those papers include, ([34],[35],[36],[6],[37],[38],[39],[40],[41],[42],[43],[44],[45],[46], and [47]). As mentioned earlier throughput is based on Shannon capacity formula which is non-linear in power allocated, so the non-linearity of the objective function could not be avoided. For example, [6] has an objective function of the form

$$
\max _{p_{k n}, x_{k n}} \sum_{k=1}^{K} \sum_{n \in N_{v}} x_{k n} \cdot u_{n} \cdot \log _{2}\left(1+p_{k n} \gamma_{k n}\right),
$$

where $u_{n}$ is the probability the subcarrier $n$ is vacant (idle), and the decision variables are $p_{k n}$ which is power level allocated to user $k$ in sub-carrier $n$ and $x_{k n}$ which is the sub-channel assignment indicator. $P_{k}$ is the power budget, $\gamma_{k, n}$ is the channel to noise ratio between $\mathrm{CR} k$ and subcarrier $n$ and $I_{k n}$ is the corresponding interference, with $I_{k n}=p_{k n} \tilde{I}_{n}$ and $\tilde{I}_{n}$ is the interference factor. The constraints are,

$$
\begin{aligned}
& \sum_{n \in N_{v}} x_{k n} p_{k n} \leq P_{k} \quad \forall k, \\
& \sum_{k=1}^{K} \sum_{n \in N_{v}} x_{k n} p_{k n} \tilde{I} \leq I_{t h}, \\
& \sum_{k=1}^{K} x_{k n} \leq 1 \quad \forall n \in N_{v}, \\
& p_{k n} \geq 0 \quad \forall n \in N_{v}, \forall k, \\
& x_{k n} \in\{0,1\}, \quad \forall n \in N_{v}, \forall k,
\end{aligned}
$$

and $I_{t h}$ is the threshold interference level.

For this problem the objective function is thus non-linear, partly because of the Shannon formula and partly due to the product form. Two sets of the constraints are also non-linear due to product form terms. What the authors did was to develop a three step heuristic algorithm in which power allocation was carried out in the first step and sub-carrier assignment in the second. This second step resulted in a Generalized Assignment problem for which there are well known heuristics. The third step involved the power allocation component. Some of the tools they used are based on their results from a previous paper [35]. Xu and Li [34] studied an RA problem in OFDMA based CRN where SUs relay data for the PUs in order to gain access to 
spectrum - a different class of problem. The authors formulated this as a Nash equilibrium problem and developed a nonlinear mixed integer programming problem for it. In order to make the problem tractable they did not consider QoS requirements. The problem is neither convex nor concave as the authors mentioned. They used a dual decomposition idea in solving the problem. Zhang and Leung [36] studied a distributed RA in OFDMA for $\mathrm{CRN}$. Their main objective was to maximize throughput while ensuring that the nominal rate requirement is met. The problem is nonlinear and formulated as goal programming. They used two algorithms to analyze the problem; one based on what they call iterative water-filling approach and the other is based on solving a dual problem. Joint optimal cooperative sensing and RA in a multichannel environment was considered by Fan et al. [48]. They formulated the problem as non-convex optimization and then proposed a bilevel optimization approach to solve it based on monotonic programming. Yu et al. [37] studied a much larger problem which involved joint optimal sensing and power allocation for a cooperative relay in CRN. Their goal was to maximize throughput and minimize outage probability. Generally their problem was non-convex and they had to develop a concave objective function equivalent for maximizing throughput and a convex equivalent problem for minimize outage probability. Optimal sensing strategy coupled with power allocation for two SUs in an AF-cooperative CRN was studied by Zhao and Kwak [38]. They showed that the resulting objective function was concave and then developed appropriate algorithm for solution accordingly. Jian et al. [39] studied a system in which cooperative relay is used to assist in transmission. Their problem formulation resulted in a nonlinear integer programming problem which they claimed had high complexity. The authors went ahead and sought a heuristic for dealing with the problem. Yao et al. [40] considered the joint optimization for downlink RA in CRN, and just like several other researchers, found the problem to be a mixed integer non-linear programming problem. They proposed a dual decomposition algorithm. Of course they had to relax the integer constraints and they were able to implement the Karush-Kuhn-Tucker conditions and then applied the ellipsoid method to update the dual variables. The approach depends strongly on the relaxation of the integer variables. Distributed RA for CRN with spectrum sharing constraints was studied by Ngo and Ngoc [41]. Even though their aim was to maximize the rate-sum capacity in the end what they did was maximize system throughput. The reality is that both sum capacity and throughput involve the Shannon capacity formula. As a result of Shannon's equation for capacity their objective function was nonlinear. And in addition they had a set of complicating constraints (spectrum sharing constraints). In summary they employed a dualization approach as other researchers in order to come up with an efficient algorithm. Lin and Lin [42] formulated joint channel allocation and power control into a mixed non-linear programming problem. By appropriately exploiting the structures of the zero-one variables they were able to convert the problem to a pure non-linear programming problem with linear constraints that resulted in convex solution space. They then used the interior point method to solve the problem. The way they exploited the structures of the zero-one variables is very clever and worth considering by other researchers. Wang et al [43] introduced overhead with route switching in their formulation of the joint spectrum allocation and power control in CRN utilizing open spectrum bands. Interference temperature constraints were also introduced. Their problem was not a classical optimization problem like others. $\mathrm{Xu}$ et al. [44], in addition to trying to achieve joint channel allocation and power control in $\mathrm{CRN}$, also incorporated fairness goal for SUs while trying to protect PU transmission. Their problem was a mixed non-linear integer programming problem. They relaxed the zero-one variables, applied Lagrangian duality and then applied the Karush-Kuhn-Tucker conditions in solving the problem. Golcezaei-Khuzani and Ardelbilpour [45] studied the RA in cognitive relaying network under the AF-protocol. The problem was a non-linear programming one and was solved based on the standard KKT conditions. Wang et al. [46] also solved the RA for CRN with cooperative relays. Just like other authors found, the problem formulation results in a mixed nonlinear programming formulation which is $N P$ hard. As a result they developed their own two stage algorithm for its analysis; the first stage involves sub channel assignment and the second one is power allocation. In a recent paper Shaat et al. [47] tried to maximize the throughput of OFDM-based cognitive two-way multiple-relay networks, by jointly optimizing power, relay assignment and subcarrier pairing. They also ended up with an MIP which they applied Lagrangian relaxation and then developed what they call a low complexity suboptimal algorithm to solve it. They did not explore the possibility of any structure that they could take advantage of.

Some papers claim to be maximizing sum capacity, which is based on the sum of several Shannon equations, and hence non-linear objective functions. Those papers include, [7],[49],[50],[51],[52],[53],[54],[55],[56],[57],[58], and [59]. We point out that even though Nguyen et al. [51] did claim that their problem is a mixed integer linear programming we feel their claim is subject to interpretation as will be discussed later. Once again, that the formulation is nonlinear is not surprising keeping in mind that sum capacity is related to the Shannon capacity formula. As an example, in [7], the authors addressed a resource allocation problem of an OFDM based CRN under the considerations of imperfect spectrum sensing, limitation of transmission power etc. The objective of this optimization problem is to maximize the sum capacities of all the SUs, and given by

$$
\max _{\rho_{k, n}, p_{k, n}} \sum_{k=1}^{K} \sum_{n=1}^{N} \rho_{k, n} \log \left(1+p_{k, n} H_{k, n}\right),
$$

where the decision variables are $p_{k, n}$ which is the allocated power on the $n^{t h}$ sub-channel used by the $k^{t h}$ SU whereas $\rho_{k, n}$ is the sub-channel allocation index. Here $H_{k, n}$ is the SNR of the $n^{\text {th }}$ subchannel used by the $k^{t h} \mathrm{SU}$ with unit power. $R_{k}$ is the rate of the $k^{t h} \mathrm{SU}$ with required rate of $R_{k}^{r e q}$, and $P_{T}$ is the transmission power limit. Let $I_{n, l}$ be the interference to the $l^{\text {th }}$ PU by an SU's access with $n^{t h}$ sub-channel with unit power and $I_{l}^{t h}$ the interference threshold of the $l^{t h}$ PU. The constraints are,

$$
p_{k, n} \geq 0, \forall n \in N, \forall k
$$




$$
\begin{aligned}
& \sum_{k=1}^{K} \sum_{n=1}^{N} \rho_{k, n} p_{k, n} \leq P_{T}, \\
& \sum_{k=1}^{K} \sum_{n=1}^{N} \rho_{k, n} p_{k, n} I_{n, l} \leq I_{l}^{t h}, l=1,2, . ., L, \\
& R_{1}: R_{2}: . .: R_{K_{0}}=\gamma_{1}: \gamma_{2}: . .: \gamma_{K_{0}}, \\
& R_{k}=R_{k}^{r e q}, k=K_{0}+1, . ., K, \\
& \rho_{k, n} \in(0,1), \forall n \in N, \forall k \\
& \sum_{k=1}^{K} \rho_{k, n}=1, \forall n \in N .
\end{aligned}
$$

In order to solve this problem the authors used a two stage approach to approximate the solution. In the first stage they carried out a sub-channel allocation to satisfy heterogeneous users' rate roughly and then removed the integer requirements constraints after that. The second stage involves power distribution among the OFDM sub-channels.

Xie, et al. [49] studied a problem that is fairly similar to the one studied by Wang et al. [7]. Even though Xie, et al. [49] also used a two stage approach to solve it, their approach is quite different. In their two stage approach, the first stage involved optimal power allocation while the second state involved channel allocation. The authors claim that the advantage with this type of approach is that it is able to track the changing radio environment in the dynamic allocation procedure. In an earlier paper Xie, et al. [50], considered the fairly similar class of problem and ensured that the minimum rate is guaranteed for SUs while proportional fairness is also incorporated. They presented a heuristic for the resulting MILNP. Nguyen et al [51] studied the joint scheduling and power allocation in a multi-cell CRN. The objective function of their optimization problem is of the form $\sum_{i} C_{i}$, where $C_{i}=\log \left(1+\gamma_{i}^{c}\right)$ and $\gamma_{i}^{c}$ is the SINR for user $i$ on channel $c$. Obviously $\gamma_{i}^{c}$ is non-linear and hence the objective function is non-linear. The authors presented a heuristic for solving the problem. Xiang et al. [52] used classical approximation approaches to solve their downlink spectrum sharing problems in CR femtocell network. The problem formulation was a mixed integer non-linear programming problem. They used a mixed primal and dual decomposition to approximate the solution by also relaxing the integer variables. As a result they decomposed the problem to those of channel allocation and power control. They efficiently applied the KKT conditions in the process. Huang et al. [53] studied the RA problem in a multi-cell CRN. Their problem is similar to those of previous researchers except that they did not seem to have considered how to explore the structure of the problem. They went ahead and sought a heuristic approach to handle the mixed integer nonlinear programming problem. Almalfouh and Stuber [54] considered the case of interference-aware power allocation for CRN with imperfect spectrum sensing. They used water filling criteria for single user CRN to carry out power allocation and applied a heuristic they developed. Choi et al. [55] studied the downlink sub-channel and power allocation in multi-cell OFDMA CRN. Their problem was also a mixed integer nonlinear programming problem. But they were able to exploit the structure of problem, as conceptually done by Xiang et al. [52], by using a dual decomposition approach. Hu and Mao [56] considered interference mitigation problem via channel assignment and power allocation for CRN based on cross-layer optimization. Surprisingly even though the original problem is that of minimizing interference it was later considered as that of maximizing throughput and then maximizing capacity. They claim to have reduced the mixed integer nonlinear programming problem to a linearized problem and presented results. Wang et al. [57] considered the problem of adaptive proportional fairness for RA of OFDM based CRN. The problem and methods used are quite similar to those discussed earlier in [7]. Du et al. [58] studied the problem of spectrum access for CR. They ended up with the same general formulation and used the KKT theorem; the problem and solution method are standard. Li et al. [59] dealt with the same type of problem as the other in this category, i.e. maximizing sum transmission rate and this resulted in mixed integer nonlinear programming problem, which was then analyzed using Lagrangian relaxation with implementation via the KKT conditions.

Other papers which are based on non-linear objective functions, and where the functions are not data rate, capacity or throughput include: [60],[61],[62],[63],[64],[65],[66], [67],[68],[69],[70], and [59].

Cumanan et al [60] considered the problem of joint fast optimal RA and beam forming in an underlay CRN. In an attempt to achieve several objectives including spectrum utilization, admitting maximum number of SUs while satisfying several constraints such as fairness, they ended up with a multi-objective optimization problem with mixed integer nonlinear programming structure. They used semidefinite programming coupled with Branch and Bound tools to analyze the problem. Shi et al. [61] considered a per node based optimal power control and multi-hop CRN, which led to a mixed integer nonlinear programming problem. They focussed on trying to optimize network performance by trying to achieve joint power control, scheduling and routing. Specifically they considered cross-layer optimization based on 3-layers and used Branch and Bound together with convex hull relaxation. Tseng et al. [62] studied the joint power control, channel assignment and routing to minimize network energy consumption while maintaining data rate requirements in a multi-hop CRN. They formulated the problem as a mixed integer nonlinear programming problem and used Lagrangian relaxation based heuristic. The authors claimed that they were able to save up to $90 \%$ energy consumption. Hou et al. [63], developed a mathematical programming problem with objective function to minimize required network wide radio spectrum resource for a set of user sessions. Their problem was set up as a mixed integer nonlinear programming problem, which was solved by using what the authors call sequential fixing procedure based on converting integer variables to achieve linear program. In trying to solve a channel selection problem in CRN with heterogeneous channel availabilities at different nodes Hou and Huang [64] presented a binary integer non-linear programming problem with the objective function given as maximizing total channel utilization. They then developed greedy channel selection (GCS) heuristic for it, which they claimed gives more than $95 \%$ close-to-optimal solution. Scheduling in a relay environment for CRN was considered by Liang and Chen [65]. They 
considered, in their model, fluctuations of usable spectrum resource, channel quality variations and interference. Their objective function was actually fractional because they were trying to maximize throughput while maintaining long-term fairness. They ended up developing a greedy heuristic to solve the problem. Tachwali et al. [66] developed a bandwidth-power product minimization problem in order to incorporate PU activity in the design of RA and limited hardware capabilities. This resulted in a mixed integer nonlinear programming problem. They decomposed the problem after using Lagrangian based approach; again a heuristic. Joint admission control and power allocation for CRN was considered by Zhang et al.[67]. Their problem was to find a subset of SUs such that the total revenue output of network is maximized in an environment where not necessarily all SUs can be accommodated. They transformed the mixed integer nonlinear problem to a smooth problem and used gradient descent based algorithm. The objective function considered by Lu et al. [68] was to maximize utility, which in this case, is used to measure QoS for users. The problem was a mixed integer nonlinear programming problem and they used the Lagrangian duality to find an approximate solution. Chen and Yuen [69] studied RA strategy for multi-user MIMO rate less-coded CRN with QoS provisioning. It resulted in a two integer product programming problem. They developed a heuristic for solving it. Guan et al. [70] considered the problem of RA for stream multimedia content in cognitive ad hoc networks with cooperative relays. They formulated the problem as joint video encoding rate control, power control, relay selection and channel assignment. This resulted in a mixed integer nonlinear programming problem. The authors used a branch and bound algorithm based on convex relaxation techniques. El-Sherif and Mohamed [71], in their paper, considered joint routing and resource allocation aiming to minimize delay in cognitive radio based mesh networks. Their problem actually is a convex optimization problem, with convex objective function and linear constraints. However, the integer valued decision variables made the problem complex.

One paper, [72] considered spectral footprint for OFDMAbased CRN and tried to minimize this. This led to a non-linear objective function, however the authors were able to decompose it to two problems; sub-channel allocation and power allocation. The sub-channel allocation problem was solved using the Hungarian method for assignment and the power allocation problem by the Lagrangian dual. Two recent papers [73] and [74] considered a hybrid underlay/overlay CRN problem. The resulting objective function was non-linear and the authors decomposed the problem into two; sub-channel allocation and power allocation. The problems were solved using cutset for the sub-channel allocation and the Lagrangian dual for the power allocation component.

Cognitive radio and femtocells are recent technology breakthroughs that aim to achieve throughput improvement by means of spectrum management and interference mitigation, respectively. However, these technologies are limited by the formerâĂŹs susceptibility to interference and the latterâĂŹs dependence on bandwidth availability. Although the deployment of cognitive femtocell networks is seen to improve the efficiency of frequency reuse and spectrum sharing, it too suffers from the challenges associated with resource management. Zhang et al. [75] proposed a near optimal cooperative bargaining resource allocation strategy that is derived based on Lagrangian dual decomposition. The optimization problem is defined by the authors as a non-convex mixed integer programming problem. The time-sharing method is used to transform the problem into a convex optimization problem; this transformed problem is regarded as a low bound of the original problem. One of the interesting contributions of this paper is the proof of the convexity of the transformed problem. Based on standard optimization techniques and the KKT conditions, the power allocation optimization problem for users in small cells are obtained. Similar to this work, is the joint sub-channel and power allocation problem which is formulated as a non-convex non-linear MIP in effort of addressing the issue of severe inter-cell interference [76]. The problem is transformed to an optimization problem with a concave objective function and convex constraints. It is then solved using the Lagrangian dual decomposition method, in which the Lagrangian dual problem is decomposed into a master problem and several subproblems that are iteratively solved.

The optimization problem associated with the spectrum selection with limited number of antennas for downlink cognitive femtocell networks was formulated by Chen et al. [77]. The optimization problem formulated aims to maximize the system's energy efficiency while being constrained by hardware limitations and user equipment. The objective function is non-linear and non-convex; and is further divided into two sub-problems: one which pertains to the spectrum selection and the other related to the power allocation. The Lagrangian dual is obtained for the power allocation optimization problem and is then evaluated using the Lagrangian algorithm. The duality gap between the primal and dual problem, which arises as a result of the nonconvexity of the objective function, is neglected as the subcarriers are shown to be large enough for the multi-carrier systems as proved in [78]. Li et al. [79] leveraged cognitive radio technology in femtocell networks and proposed an asymptotically optimal resource allocation algorithm using dual decomposition methods. The authors considered open access OFDMA femtocell networks for the scenario where a macrocell user performs handover from its serving macrocell base station to a nearby femtocell access point. As was performed by Chen et al. [77], a joint subchannel and power optimization problem is formulated and an efficient algorithm using dual decomposition methods is proposed.

Zhang et al. [80] proposed a power management scheme for adjacent femtocell networks to reduce interference while satisfying capacity requirements. The authors considered the distance-dependent variation of channel gain in the analysis of network interference and sum capacity. In order to maximize the sum capacity, a non-convex power optimization problem is formulated. This non-convex problem is then divided into two convex problems which is solved by Lagrangian dual theory and linear programming methods. A multi-objective optimization problem with mixed integer variables for the joint power control, base station assignment, and channel assignment scheme in cognitive femtocell networks is formulated by Torregoza et al. [81]. The multiobjective optimization problem aims to maximize the achievable throughput of the system while minimizing the need for femtocell compensation. 
The classification of the objective function into linear or nonlinear gives us a quick overview of the possible ratio of papers that have the potential of being linearly based. Clearly for a problem to be linearly based, in addition to linear objective function, it must also have linearly based constraints. Seeing that of the 79 papers surveyed only 16 have linearly based objective function, the number of linearly based problems is definitely less than or equal to 16 . As for non-linearly based objective functions we know that the problem will be a non-linear programming one. It is known that there are useful tools for solving convex optimization problems (see [8]). However, only a very limited number of authors declared whether their nonlinear programming problems are convex or non-convex. Most went straight for heuristics or first declared that their problem is NP-hard or complex and then using heuristics or special techniques. In that case we assume that their problem is non-convex. As such most of the non-linear based problems encountered in the survey can be declared as being non-convex programming problems, especially if there are zero-one variables involved then they are non-convex.

\section{SURVEY OF TOOLS COMMONLY USED FOR SOLVING RA PROBLEMS FOR CRN}

Unless a problem fits nicely into a well-known and well researched class of optimization problem, we usually have to carry out some form of approximation or find some bounds. In this section we discuss the commonly employed techniques and identify which of them were used in some papers. We also discuss how effective those techniques were in each case.

\section{A. Exploring and Exploiting Problem Structure}

One of the tools commonly used when mathematical methods are applied to practical problems is that of exploiting a special structure that may exist to assist in making the problem easier. Once the special structure is found, if it exists, then special techniques are employed to solve the problem. Here we discuss two very interesting cases in which the authors found and exploited a special structure discovered.

The first one is the work of Shu and Krunz [16]. In that paper, the authors addressed the issues of coordinated channel access as a joint power control, rate control and channel assignment optimization problem. The objective of this optimization problem is to maximize all of the sum rate of all SUs over all channels. The objective function is,

$$
\max _{x_{i}^{(m)}, P_{i}^{(m)}} \sum_{i=1}^{N} \sum_{m=1}^{M} x_{i}^{(m)} R_{i}^{(m)},
$$

where the decision variables are, $x_{i}^{(m)}$ which signifies binary channel allocation indicator and $P_{i}^{(m)}$ which is the transmission power allocated on channel $m$. Note that the transmission rate $R_{i}^{(m)}$ is a function of $P_{i}^{(m)}$. The constraints are,

$$
\frac{1}{h_{i}^{(m)}}\left(q_{D(i)}^{(m)}+N_{0}\right) f^{-1}\left(r_{i}^{(m)}\right) \leq \hat{P}_{i}^{(m)} \quad(m=1,2, . ., M),
$$

$$
\begin{aligned}
& \sum_{m=1}^{M} \frac{1}{h_{i}^{(m)}}\left(q_{D(i)}^{(m)}+N_{0}\right) f^{-1}\left(r_{i}^{(m)}\right) \leq P_{(\max , i)}, \\
& x_{i}^{m}+x_{j}^{m} \leq 1, \quad \forall j \in I_{i}^{(m)},
\end{aligned}
$$

where

$$
R_{i}^{(m)}=B_{m} f\left(\frac{P_{i}^{(m)} h_{i}^{(m)}}{q_{D(i)}^{(m)}+N_{0}}\right),
$$

and $f$ is any arbitrary rate-SINR function, $N_{0}$ is the AWGN and $q_{D(i)}^{(m)}$ is received interference over channel $m$ at $D(i)$. The other parameters are; $h_{i}^{m}$ the channel gain of link $i$ on channel $m$, $P_{(\max , i)}$ the maximum battery power, $r_{i}^{(m)}$ the spectrum efficiency of link $i$ on channel $m$, and $I_{i}^{(m)}$ the set of interfering CR links.

The first constraint implies that transmission power of link $i$ on channel $m$ cannot exceed the threshold level of power mask, $\hat{P}_{i}^{(m)}$. The second constraint implies that the sum of transmission power over all the channels cannot exceed the maximum power level. The third constraint denotes channel allocation policy. This policy implies that if channel $m$ is being used by link $i$, then it cannot be used by any other link that will interfere with link $i$ on channel $m$ and vice versa.

This optimization problem is an MINLP problem which is generally NP-hard. To find a solution, the authors cleverly explored the special structure of the problem. They observed that actual communication systems only support a finite set of discrete transmission rates. They were able to exploit this special structure to convert this MINLP problem into a binary integer programming (BIP) problem. This BIP problem contains only binary variables and linear objective function along with the constraints. This transformation also applies to a given data rate and SINR relationship. The authors have proposed a centralized polynomial time linear programming with sequential fixing (LPSF) approximate algorithm for solving the BIP problem. The authors claimed that the accuracy of their proposed algorithm has been proved through their numerical examples.

It is true that several of the optimization problems encountered in telecommunication systems, especially wireless and in particular CRN, are complex and usually of the mixed-integer NLP types which are often NP-hard. However additional efforts to seek special structure can sometimes lead to finding more manageable solution techniques.

A second example is the work of Salameh [29] in which the objective function looks closely like that of [16], even though the constraints are not entirely the same. The problem is a mixed-integer NLP and Salameh [29] also exploited about the same type of structure observed in [16] and used it to convert the problem to a mixed-integer LP

Shin, et al. [32] in their work converted a non-convex optimization problem to a geometric programming problem, which in turn is converted to a convex programming problem. This is a "double-conversion", but the main thing is that it allowed the problem to be cast in a format that is standard - convex programming. 
Suppose the problem being considered is linear where

$$
f(\mathbf{x}, \mathbf{y})=\mathbf{c}\left[\begin{array}{l}
\mathbf{x} \\
\mathbf{y}
\end{array}\right]
$$

where $\mathbf{c}$ is a row vector, and also $\mathbf{g}(\mathbf{x}, \mathbf{y})$ is linear, i.e. can be written as $A\left[\begin{array}{l}\mathbf{x} \\ \mathbf{y}\end{array}\right]$ and supposing the problem is the size of matrix $A$. Some authors [82], [83] were able to find some special structures in $A$ and used column generation [84], [85], a special technique that can capitalize on such structures. Even though this technique has been used in some telecommunications research its application in RA for cognitive radio networks is limited.

\section{B. Linear Programming}

Suppose the problem falls into the Linear Programming category, i.e. Eq. (1) and Eq. (2) are linear and Constraint (4) is simply a non-negativity constraint and does not have integer requirements, then we may employ the well known simplex algorithm or the interior point method [86], [87]. However, the chances of the problem falling exactly into that category are very rare. Usually there is a non-linear component and integer variables. Assuming that the non-linear functions can be well approximated by linear functions, one may apply mixed integer programming methods which will be based on Branch and Bound approach and is discussed in Section V.C. Such problems can then be solved using LP techniques and the results obtained, if feasible, become bounds for the true solutions. One example in which this approach was adopted is Shi and Hou [13]. They linearized the non-linear constraint, which was the only non-linear function in the problem and then relaxed the integer variables. So they obtained some bounds for the problem. Such bounds are usually not quite tight. Meanwhile we focus on the cases where the integer variables can be relaxed, i.e. allowed to assume non-negative real values. Sometimes researchers have then taken the resulting non-negative real values of the variables and rounded up or down to give approximate solutions. In another paper $\mathrm{Hu}$, et al. [56] applied the linear programming relaxation to an integer linear programming problem. Zhang et al. [80] transformed their initial non-convex problem formulation into a convex problem by changing the optimized variable and ignoring some constraints, this is then solved by the Lagrangian dual method. After this, the authors re-consider the constraints ignored in the first step and formulate a linear programming problem to obtain the final solution.

\section{Branch and Bound}

Branch and Bound is a tool that is applicable to either linear or non-linear programming problems that are integer or mixedinteger based, even though it has been popularly used more often for ILPs and MILPs. Essentially it first relaxes some of the constraints of the problem and solves it. If the solution is feasible for the original problem, then that is the optimal solution. Otherwise the solution provides a kind of bound and the problem then branches by splitting the problem solution space into two separate ones (usually by adding new constraints to break some of the violating constraints). This process results in a tree with branches of solution space created at each step. Through such a process a form of feasible solution is obtained. For a detailed treatment of Branch and Bound see [88]. For most RA problems the integer component is first relaxed and based on that a solution is obtained. If the integer conditions have been met then this solution is the optimal solution desired. Usually the integer variables have been violated then this solution is only a bound to the original problem. The next stage is then to partition the solution space into two, based on one of the violated integer constraints selected. This is a branch point and at least one of the two problems resulting (at this node) is solved. The process is repeated at each of these nodes until a feasible solution to the original problem is obtained. This solution then serves as a true bound. This continues until an optimal solution is obtained or, in a more realistic situation, until some feasible solutions to the original problem have been obtained and the best of them serves a bound. It also provides the gap between it and the relaxed one used as a measure of quality of the solution. The use of Branch and Bound has been very popular in this class of problems. Rahulamathavan, et al. [11] reformulated their MINLP to an ILP and used Branch and Bound method to solve it. The decision variable in their problem is $c_{k, n}$, which is the number of bits allocated to the $k^{t h}$ secondary user in the $n^{t h}$ sub-carrier. Other papers that have applied Branch and Bound include, [18], [60] and [12]. The problem with branch and bound method is that one never knows how long it would take to get a very good bound. For example, Mitran et al. [12] found that for their MILP problem, even though Branch and Bound is a good candidate technique to use, its behaviour was inconsistent. For some example problems the authors obtained exact optimal results within a time limit and in other cases the Branch and Bound ran until its time limit and in some cases it stopped because the enumeration tree became too large, i.e. there were too many branches. This is understandable, given that there is a function $f(z)$ in one of the constraints for which it is not clear how it is found, and its linearity is not established. In another later paper, Mitran et al. [26] studied a closely related problem which includes what they term queue-awareness. In that case the optimization problem became a very large non-linear integer programming problem and they had to develop heuristics to solve it. However, Shi et al. [61] and Guan et al. [70] did use Branch and Bound in a creative manner by complementing it with what they call convex relaxation, an approach that creates a form of linear relaxation in conjunction with Branch and Bound. Torregoza et al. [81] used the branch and bound method for solving their nonconvex problem in a predetermined time for a fixed number of variables.

Branch and Cut is a technique that combines Branch and Bound with some elements of Cutting Plane [89]. It has potential of working well in some instances where Branch and Bound is taking too long. When a problem is well suited to Branch and Bound, but using it leads to inconsistent results or the algorithm is taking too long to complete, one could consider Branch and Cut. A candidate problem to revisit using Branch and Cut is the one in [12].

\section{Lagrangian Duality}

Lagrangian duality, as mentioned earlier, is based on dualizing all the major constraints, i.e. other than the non-negativity 
constraints, and making sure that the integer constraints are relaxed if they are part of the problem. The problem is then solved using the classical KKT conditions, on the assumption that the relaxed problem is a convex programming problem. Several papers actually applied this technique, among them is [5], even though their problem is a non-convex one and they claim that they were able to obtain an optimal solution. Other papers include: [28],[40], [41],[43],[44],[49],[58],[68], [45],[59],[75],[76],[77],[79], and [80]. El-Sherif and Mohamed [71] used this technique to obtain sub-optimal solution to their problem. They relaxed the integer variables in order to pursue this solution method.

\section{E. Lagrangian Relaxation}

Lagrangian relaxation is a tool made popular by Fisher [9], [10] and Geoffrion [90]. It involves dualizing some of the constraints of an integer linear programming problem. This usually entails removing some of the constraints to make the problem easier to work with. An initial value for the dual variables for those constraints are selected and used in the optimization problem; those values are usually not good estimates initially, but through an iterative process the values are adjusted and they usually converge for well behaved problems. Results from this always serve as good bounds for the original problem. The challenges with this method are usually deciding on which constraints to dualize and how to select initial values of the dual variables. Given the power of this method, it is surprising that it is not used more often for this class of problems. Generally researchers seem to gravitate towards the Lagrangian duality, without first assessing the possibility of using Lagrangian relaxation. The key difference is that Lagrangian relaxation (LR) and Lagrangian duality (LD) can be summarized in the following. Consider a simple non-linear integer programming problem which is to maximize $z=f(\mathbf{x})$, s.t. $\mathbf{g}(\mathbf{x}) \leq \mathbf{b}_{1}, \mathbf{d}(\mathbf{x}) \leq$ $\mathbf{b}_{2}, \mathbf{x} \geq 0, \mathbf{x} \in I^{k}$, where $\mathbf{x}$ is a vector of $k$ integer variables. We let the number of constraints in $g(\mathbf{x}) \leq \mathbf{b}_{1}$ be $n$ and for $\mathbf{d}(\mathbf{x}) \leq \mathbf{b}_{2}$ be $m$. The LR of this problem, which we call $L R(u)$ could be, for example

$$
\begin{gathered}
z(\mathbf{u})=\max \left\{f(\mathbf{x})+\mathbf{u}^{T}\left(\mathbf{b}_{2}-\mathbf{d}(\mathbf{x})\right) ; \mathbf{g}(\mathbf{x}) \leq \mathbf{b}_{1},\right. \\
\left.\mathbf{x} \geq 0, \mathbf{x} \in I^{k}\right\} .
\end{gathered}
$$

We want to find $u$ that gives an optimal and feasible solution to this. The Lagrangian duality of this is given as

$$
Z_{L D}=\min \left\{z(\mathbf{u}) ; \mathbf{u} \geq 0, \mathbf{u} \in R^{m}\right\} .
$$

The solution to this problem is an upper bound to the $z(\mathbf{u})$, i.e. the LR. If in the solution of LR we obtain integral $\mathbf{x}$ then the $z_{L D}$ is the value of the LR. Often researchers relax the integrality of $\mathbf{x}$ in LD and then apply the KKT conditions as discussed earlier. As one can see, the LD is a special case of the LR.

\section{F. Heuristics}

Heuristics are generally seen as techniques that are based on logical ideas on how to improve solutions and are problem specific. It is not quite common to find heuristics that can be generalized to a group of problems. However they usually do work very well for the problems that they are designed for. Some examples of problems in which heuristics were used include: [26], [35], [6],[20], [51], [52], [50], [53], [54], [57], [65],[69] and [39] who all developed problem specific heuristics. Guo et al. [19] developed a heuristic which they described as a greedy algorithm. Actually, it is a heuristic in the same sense as the others. In another paper Wang, et al. [7] considered an MIP and after eliminating all possible classical methods and approximations that can be used to solve it, they resorted to a heuristic method. Hoang et al. [23] and Hoang et al. [22] formulated their problem as a form of mixed-integer linear programming problem. They went ahead and developed what they call a sub-optimal technique - a heuristic - to solve the problem. It is surprising that despite considering the structure of the problem they only ended up with a heuristic instead of a standard approach. Hou et al.[64] first proved that the binary NLP they had was definitely NP-hard before embarking on seeking a greedy algorithm.

Metaheuristic methods are those that are based on genetic algorithm, simulated annealing, tabu search, ant colony, particle swarm, etc., i.e., those that are developed based on some natural occurring situations. Waheed et al. [27] used both swarm optimization and genetic algorithm. These methods seem to work for each problem studied, but the idea or knowledge gained are usually not quite transferable to other problems.

\section{G. Other Methods}

Xie et al. [31] modelled their system using stochastic programming approach and later solved it as a discrete stochastic programming problem. Shin et al. [32] applied geometric programming tool, which is a well established approach. In a special case, Lin et al. [42] used the interior point method, a technique that is an alternative to the simplex method, but can also be used for non-linear programming problems, especially if integer constraints are not an issue. Wang et al. [21] formulated their problem as a binary linear program and designed an algorithm called directional search to handle the problem. Another very interesting technique is the one used by Fan et al. [48] to handle a non-convex problem. They call it bilevel optimization and monotonic program. Zhao et al. [38] used a classical approximation method to deal with their problem which is the maximization of a concave function and the constraints are well behaved. Zhang et al. [36] used goal programming combined with a heuristic. Zhang et al. [67] on the other hand relaxed the integer constraints and then applied the gradient descent based method to solve the problem. This method is a classical approach used for non-linear programming problems. Tachwali et al. [66] created an iterative method based on decomposition to handle their mixed-integer NLP. An et al. [25] used a lowcomplexity iteration algorithm based on gradient-assisted binary search algorithm for solving the resource management problem.

Wang et al. [46] used barrier method and speedup Newton method to deal with their mixed-integer programming problem resulting from maximizing overall system throughput. The authors claim the problem was intractable and thus developed a two-stage method to deal with it

Knapsack problems can be solved using different approaches, including Branch and Bound and dynamic programming, but greedy algorithms have been applied in many cases. Zhang et 
al. [30] set their problem up as a knapsack problem and used greedy algorithm for the solution.

Some papers simply set up the optimization problem, did not seek special structures but went ahead and used known software packages to seek solutions. For example, in [14] and [15], the authors set up their problems as mixed-integer programming problems and used the software LINGO to obtain solutions.

Table 1 provides a summary of tools used to solve the resource allocation problems as found in the literature.

\section{CHALLENGES AND OPEN PROBLEMS}

There are several ways to carry out approximations and there are also issues associated with applying existing knowledge for RA. In this section we discuss such issues, present the challenges and suggestions.

\section{A. Linearization}

One that leads the way is the linearization type of approximation. Some expressions are commonly encountered in this RA class of problems. If they could be properly linearized to be close to the values obtained from the original expression within a particular limit and bounds that would make solving this class of problems much easier. Consider the expression

$$
R_{m}=\sum_{k=1}^{K} a_{m k} \log _{2}\left(1+\frac{h_{m k} P_{m k}}{\Gamma \sigma_{k}^{2}}\right), \forall m .
$$

This type of expression appears in most RA problems for CRN. Other than the $a_{m k}$ component of the expression the rest is based on the well known Shannon limit and it is non-linear in $p_{m k}$. It will appear and re-appear in constraints and sometimes in the objective functions as long as we are concerned about capacity and data rates.

Consider a function

$$
f(x)=\log _{2}(a+b x) .
$$

We can study its first order Taylor approximation and see when and within what ranges it does represent the original function well. If we can do this we might be able to convert some nonlinear programming problems to LP or quadratic programming. We also know that second order Taylor approximation would probably be better even though that takes us into the quadratic optimization class of problems, if they do not involve product forms. It is well known that quadratic programming has better potential of getting solved effectively than general NLP. With recent developing interest in semi-definite programming such an undertaking may be worthwhile.

Shi and Hou in their paper [13] presented a linearizing approximation method for the function $\log _{2}\left(1+\frac{a}{b c} x\right)$. Let $d=b c$ and specifically consider a variable $x$ and the expression

$$
\log _{2}\left(1+\frac{a}{d} x\right)
$$

The authors proceeded by defining a variable $v$ which replaces the expression and then applied what they call three tangential supports as the approximation which they say is a convex envelope linear relaxation, thereby leading to four constraints instead of one. Their approximation is not based on Taylor series. What they did was to replace the expression in (16) with four inequalities as follows:

$$
\begin{gathered}
v-\frac{a}{d} x \leq 0 \\
v-\frac{a}{(d+a \beta) \ln 2} x \leq \log _{2}\left(1+\frac{a \beta}{d}\right)-\frac{a \beta}{(d+a \beta) \ln 2}, \\
v-\frac{a}{(d+a P) \ln 2} x \leq \log _{2}\left(1+\frac{a P}{d}\right)-\frac{a \beta}{(d+a P) \ln 2},
\end{gathered}
$$

$$
v-\frac{\log _{2}\left[1+a \frac{P}{d}\right]}{P} x \geq 0
$$

where $P$ is a known constant and in their case it is the maximum power, and $\beta=\frac{A}{B}$ with

$$
A=\left[\log _{2}\left(1+\frac{a P}{d}\right)-\frac{a P}{(d+a P) \ln 2}\right],
$$

and

$$
B=\left[\frac{a}{d \ln 2}-\frac{a}{(d+a P) \ln 2}\right] .
$$

Whereas the approximation for linearization itself is cumbersome to use because it introduces three additional constraints, the idea is in the right direction. Efforts should be made to improve on this work.

Li et al. [33] also used a clever linearization technique. First they noticed that their formulation is in the linear form except for one of their constraints which captures transmission rate between two nodes. This allowed them to develop an effective approximation method for solving the problem.

\section{B. Dealing with Fractional Constraints}

One class of constraints frequently encountered is the one to do with minimum SNR requirements for QoS. This is usually of the form

$$
\frac{g_{j} p_{j}}{K+\sum_{\forall k \neq j} g_{k} p_{k}} \geq \gamma
$$

where $g_{j}$ and $p_{j}$ are the gain and power from user $j, K$ is some constant usually representing thermal and other factors, and $\gamma$ is the minimum SNR required by the users in order to meet the QoS. This constraint is of the fractional type and does create a challenge when it also appears in the objective function; otherwise as a constraint only it can be linearized. Often, however, when it appears it is usually as a constraint and also as part of an objective function. Efforts should be directed towards finding reasonable approximation to this function that makes it easier to manage. For example in a different type of problem, Abadpour et al. [91] were able to take advantage of the structure presented by their problem to come up with a nice replacement format for this function that made it easier to work with. 
Table 1. Summary of tools used to solve RA problems found in literature

\begin{tabular}{|l|l|l|}
\hline & Linear Objective Function & Non-linear Objective Function \\
\hline \hline $\begin{array}{l}\text { Exploring and Exploit- } \\
\text { ing Problem Structure }\end{array}$ & {$[16]$} & {$[7],[29],[32],[55]$} \\
\hline Linear Programming & {$[13],[16],[18],[24]$} & {$[5],[80]$} \\
\hline Branch and Bound & {$[11],[12],[17]$} & {$[60],[61],[70],[81]$} \\
\hline Lagrangian Duality & & {$[5],[28],[40],[41],[43],[44],[45]$,} \\
& & {$[49],[58],[59],[68],[71],[72]$,} \\
& & {$[73],[74],[75],[76],[77],[79]$,} \\
\hline Lagrangian Relaxation & & {$[80]$} \\
\hline Heuristics & {$[19],[20],[22],[23]$} & {$[6],[26],[27],[33],[34],[35],[39]$,} \\
& & {$[50],[51],[52],[53],[54],[57]$,} \\
\hline Other Methods & {$[64],[65],[69]$} \\
& {$[14],[15],[21],[25]$} & {$[30],[31],[32],[36],[37],[38]$,} \\
& & {$[42],[46],[48],[63],[66],[67]$,} \\
& {$[72],[73],[74]$} \\
\hline
\end{tabular}

\section{Effective Use of Lagrangian Relaxation}

As pointed out earlier, LR was made popular by Fisher [9], [10] and Geoffrion [90]. Specifically, it dualizes some of the constraints, in ILPs and MILPs, which when not in the set of constraints may make solving the problem easier or provide special structures. For example, consider the problem of Section II. We assume that $f(\mathbf{x}, \mathbf{y})$ and $\mathbf{g}(\mathbf{x}, \mathbf{y})$ are all linear. Suppose Eq. (2) can be partitioned into two subsets and written as

$$
\begin{aligned}
& \text { s.t. } g_{i}(\mathbf{x}, \mathbf{y}) \leq b_{i}, i=1,2, \cdots, r_{1}, \\
& g_{i}(\mathbf{x}, \mathbf{y}) \leq b_{i}, i=r_{1}+1, r_{1}+2, \cdots, r
\end{aligned}
$$

and the situation is such that without the first $r_{1}$ constraints, the problem may have a structure that makes it easier to solve. We may then write a new problem as follows

$$
\begin{gathered}
\min _{\mathbf{x}, \mathbf{y}} f(\mathbf{x}, \mathbf{y})+\sum_{j=1}^{r_{1}} \lambda_{j}\left(b_{j}-g_{i}(\mathbf{x}, \mathbf{y})\right) \\
\text { s.t. } g_{i}(\mathbf{x}, \mathbf{y}) \leq b_{i}, i=r_{1}+1, r_{1}+2, \cdots, r, \\
x_{k} \geq 0, \quad k=1,2, \cdots, n, \\
y_{j} \in \mathcal{I}, \quad j=1,2, \cdots, m . \\
\lambda_{j} \geq 0, \quad j=1,2, \cdots, r_{1},
\end{gathered}
$$

and the values of $\lambda_{j}$ are estimated based on the knowledge of the problem and those values are adjusted after each iteration until a very good bound has been found.

This approach helps tremendously if one is able to identify such a set of constraints that can be dualized. The common challenges with this approach are:

1. identifying the most appropriate constraints to dualize,
2. selecting the initial dual variables corresponding to those constraints,

3. determining how to adjust the values of those dual variables at each iteration. Even though the sub gradient method was suggested in [9], in some instances we have found more efficient approaches [92].

Efforts should be made to determine how to make progress in this area regarding optimization problems for RA in cognitive radio networks. Some of the constraints appear commonly in this class of problems and theoretical research efforts should be pursued in this respect.

\section{Decomposition Algorithms}

Decomposition algorithms attempt to capitalize on some special structures of the problem, and then decompose it to several smaller problems that become manageable. As an example, consider the following. Suppose Eq. (1) can be written as

$$
\min _{\mathbf{x}, \mathbf{y}} z=f_{1}(\mathbf{x})+f_{2}(\mathbf{y})
$$

and Eq. (2) written as

$$
\begin{gathered}
g_{i}(\mathbf{x}) \leq b_{i}, \quad i=1,2, \cdots, k \\
g_{i}(\mathbf{y}) \leq b_{i}, \quad i=k+1, k+2, \cdots, r-1,
\end{gathered}
$$

and

$$
g_{r}(\mathbf{x}, \mathbf{y})=g_{r 1}(\mathbf{x})+g_{r 2}(\mathbf{y}) \leq b_{r} .
$$

We could use a relaxation approach and transform the problem to

$$
\begin{gathered}
\min _{\mathbf{x}, \mathbf{y}} z_{L}=f_{1}(\mathbf{x})+f_{2}(\mathbf{y})+\lambda_{r}\left[b_{r}-g_{r 1}(\mathbf{x})+g_{r 2}(\mathbf{y})\right] \\
g_{i}(\mathbf{x}) \leq b_{i}, \quad i=1,2, \cdots, k
\end{gathered}
$$




$$
g_{i}(\mathbf{y}) \leq b_{i}, \quad i=k+1, k+2, \cdots, r-1,
$$

plus the standard non-negativity and appropriate integer constraints, where $\lambda_{r}$ is the associated dual variable to be determined.

It is clear that the objective function can now be written as two loosely independent parts with the only connecting parameter being the dual variable $\lambda_{r}$ which can be used effectively in assessing the convergence of any algorithm implemented for the problem. Hence this problem can be decomposed into two separate problems that are, in theory or superficially, independent, as follows

$$
\begin{gathered}
\text { Problem } P_{1}: \\
\min _{\mathbf{x}} z_{L}(1)=f_{1}(\mathbf{x})-\lambda_{r} g_{r 1}(\mathbf{x}) \\
\text { s.t. } g_{i}(\mathbf{x}) \leq b_{i}, \quad i=1,2, \cdots, k,
\end{gathered}
$$

and

$$
\begin{gathered}
\text { Problem } P_{2}: \\
\min _{\mathbf{y}} z_{L}(2)=f_{2}(\mathbf{y})-\lambda_{r} g_{r 2}(\mathbf{y}) \\
\text { s.t. } g_{i}(\mathbf{y}) \leq b_{i}, \quad i=k+1, k+2, \cdots, r-1 .
\end{gathered}
$$

Now each of these can be solved individually and the solutions combined later. This general idea was used by [55]. Xiang et al. [52] also used a different type of decomposition which is referred to as the Primal-Dual decomposition. Xu et al. [34] found that their problem was not a convex optimization (the objective function was neither convex nor concave) but that by using the idea of dual-decomposition the problem became manageable.

There are other well established decomposition tools such as Bender's decomposition which are very suitable to some class of non-linear problems that are decomposable or near decomposable and the Dantzig-Wolfe decomposition which is more appropriate for linearly based optimization and does use Column Generation tools partially. Both Dantzig-Wolfe decomposition and Column Generation are mainly for linear programming based problems. Such types of problems rarely occur in RA for CRN, except in the few cases mentioned earlier and even then are approximations. However, Benders decomposition originally developed in [93] for linear program based problems was later extended to non-linear based problems by Geoffrion [94]. Benders decomposition can be used also for stochastic programming problems. We briefly present Bender's decomposition for solving mixed integer nonlinear programming problem. We adapt the presentation approach in [95].

We present Bender's decomposition for mixed integer nonlinear programming problem. Consider the problem presented in Eq. (1) to Eq. (4), and for simplicity without loss of generality we make $\mathbf{b}=\mathbf{0}$. Let the problem be re-written in the following form:

$$
\min _{\mathbf{x}, \mathbf{y}} z=f(\mathbf{x}, \mathbf{y})
$$

$$
\text { s.t. } \mathbf{g}(\mathbf{x}, \mathbf{y}) \leq \mathbf{0}
$$

$$
\mathbf{x} \in \Re^{n},
$$

$$
\mathbf{y} \in \mathcal{Z}^{m}
$$

On the assumption that $f(\mathbf{x}, \mathbf{y})$ and $\mathbf{g}(\mathbf{x}, \mathbf{y})$ are convex with respect to $\mathbf{x}$, then for a fixed $\overline{\mathbf{y}} \in \mathcal{Z}^{m}$ the problem can be decomposed into two subproblems; 1) Primal and 2) Master as follow:

\section{Primal}

$$
\begin{aligned}
& \min _{\mathbf{x}} z=f(\mathbf{x}, \overline{\mathbf{y}}) \\
& \text { s.t. } \mathbf{g}(\mathbf{x}, \overline{\mathbf{y}}) \leq \mathbf{0}
\end{aligned}
$$

$$
\mathbf{x} \in \Re^{n},
$$

Master

$$
\begin{aligned}
& \min _{\mathbf{y} \in \mathcal{Z}^{m}} \max _{\mathbf{u} \in \Re^{r}}\left[\min _{\mathbf{x} \in \Re^{n}}\left\{f(\mathbf{x}, \mathbf{y})+\mathbf{u}^{T} \mathbf{g}(\mathbf{x}, \mathbf{y})\right]\right] \\
& \text { s.t. } \min _{\mathbf{x} \in \Re^{n}}\left\{\lambda^{T} \mathbf{g}(\mathbf{x}, \mathbf{y})\right\} \leq \mathbf{0}, \quad \forall \lambda \in \Lambda,
\end{aligned}
$$

where $\Lambda=\left\{\lambda \geq \mathbf{0}, \quad \sum_{i} \lambda_{i}=1\right\}$.

This is just one form of the Master. Geoffrion [94] wrote it in a slightly different way. One then solves the Primal and master alternately until convergence or desired gap is achieved. The details of how to implement the decomposition can be found in the references cited in [94].

The idea of decomposition is an area that needs to be studied more in the future in terms of optimization for RA in cognitive radio networks. These techniques have great potential of making some problems less complex to analyze.

Few papers in the literature have used some versions of decompositions. For example, [72],[73], and [74] have all used some form of decomposition, to mention just few. The most common type of decompositions we came across in the literature involve decomposing the problem into 1) power allocation and 2) sub-channel allocation. Occasionally the power allocation component would have to be further approximated by, may be Lagrangian dual problem. In any case it makes the problem manageable.

\section{E. Column Generation}

This is another technique that has great potential when it is applied in appropriate problems. It was briefly discussed in Section V(A), as an example of a technique that explores and exploits special structures. It has been used more commonly for optimization problems with linearly based constraints, usually linear programming which have some special structures associated with matrix $A$ of the constraints. For example, consider the 
case where all functions in Eqs (1) and (2) are linear, and for simplicity let us write them as classical MILP of the form

$$
\begin{gathered}
\max _{\mathbf{x}, \mathbf{y}} z=\mathbf{c}\left[\begin{array}{l}
\mathbf{x} \\
\mathbf{y}
\end{array}\right] \\
\text { s.t. } \mathbf{A}\left[\begin{array}{l}
\mathbf{x} \\
\mathbf{y}
\end{array}\right] \leq \mathbf{b}, \\
x_{k} \geq 0, k=1,2, \cdots, n ; y_{j} \in \mathcal{I}, j=1,2, \cdots, m .
\end{gathered}
$$

Here $A$ is a matrix associated with the constraints. There are some instances when obtaining the matrix $A$ could be onerous, either because it is too large or hard to generate all the columns. A very good example of where this can be found is in cutting stock problems [84] and [85]. Suppose it is laborious to find all the columns of $A$ because it is large. If there is a structure or a pattern associated with $\mathbf{A}$ such that for each column $\mathbf{a}_{j}$ of the matrix $\mathbf{A}$ there is a row vector $\mathbf{d}_{j}$ and a constant $r_{j}$ such that $\mathbf{d}_{j} \mathbf{a}_{j} \leq r_{j}$, then we can use the idea of the simplex algorithm to determine the entering column (variable) by finding which variable $j$ minimizes $\mathbf{c}_{B V} B^{-1} \mathbf{a}_{j}-c_{j}$, subject to $\mathbf{d}_{j} \mathbf{a}_{j} \leq r_{j}$. Here $\mathbf{c}_{B V}$ is the vector of the coefficients, in the objective function, of the current basic solution, $B$. The matrix $B$ is the associated portion of matrix $\mathbf{A}$ and $c_{j}$ is the objective function coefficient associated with variable $j$.

This idea was used in a communication problem by [83] for scheduling and power control for vertical spectrum in STDMA wireless networks. The use of this technique for resource allocation problems in cognitive radio networks should be explored more. The original papers that introduced column generation for solving communication problems are [84] and [85].

As a matter of fact, the Dantzig-Wolfe decomposition algorithm does use some aspects of column generation in its scheme. We believe that column generation applications to complex RA problems in CRN have not received as much attention as they should.

Finally, we believe that trying to find ways of managing complexity in MIP is one of the keys to effectively dealing with RA issues for CRN. To that we suggest exploring the idea of fixed or constant number of variables proposed in [96] and [97].

\section{CONCLUSIONS}

In this paper we carried out a survey of papers that developed methods for RA in CRN and observed that most of the optimization problems encountered did not fit into the classical convex programming problems. Hence classical and conventional methods available in the literature could not be used for solving them. Several authors have been creative in how they used the knowledge of their problems together with the classical methods in order to come up with good solutions. We also pointed out the challenges in this class of problems, summarized what we classify as open problems and suggested some research directions in this area.

It is clear that because several allocation processes lead to a decision variable that is binary in nature, i.e. assign a channel or not, a zero-one programming is common to nearly all RA problems in addition to power allocation which is a real variable. Others involve deciding on the number of channels to assign which is integer and/or power allocation, which is not integer. Hence most of the problems are in a form of mixed integer programming, which are usually solved by Branch-and-Bound, cutting plane method, or problem based heuristics. Generally, the first of these techniques is more commonly used. Branchand-Bound, even though sometimes computationally challenging, does provide very good and well founded bounds and are thus more scientifically preferred. However, very often heuristics which are developed based on the knowledge of the specific problem are usually easier to implement and do sometimes give good results, as can be observed from the literature. Our suggestion here is that one may pursue a heuristic initially, if necessary, and then quickly develop a branch-and-bound result to assess the quality of the heuristic for each example.

Generally, if all the problems were of ILP types then the standard approach would be to apply some form of relaxation, either LR or LP relaxation. With LP relaxation, we are only able to obtain bounds, we would still need to solve the ILP using some other method. However with the LP relaxation we would be able to obtain a bound very quickly. With Lagrangian Relaxation, we try to dualize some of the constraints. This method gives us acceptable solutions and their bounds. The challenges with this approach usually are: 1) deciding on which constraints to dualize and 2) selecting the starting dual variables to use in the problem. These two challenges, in our opinion, form a basis for one of the open problems in this field. Selection of which constraints to dualize should be based on the structure of the problem and also knowledge of the features of the problem. Secondly, selecting the initial values of the dual variables can be achieved based on the importance of a constraint and to what extent one thinks or wants it to be binding. Coming up with a unified approach for dealing with these two issues will go a long way to improving methods for RA for CRN. Some problems have been solved by applying Lagrangian Duality and then using the KKT based tools. While these methods are well founded scientifically one has to search for special tools for the computational component of the solution.

The three key directions we feel researchers should try and focus on in looking for efficient and effective solutions are: LR, Decomposition methods, and Linearization of functions to help turn CRN problems to LP types. However, most important is that researchers should take the time to explore possibilities of useful structures in formulated problems before embarking on just developing heuristics.

\section{ACKNOWLEDGMENT}

The authors would like to thank the anonymous referees whose comments have helped us improve the presentation of the paper. This research was supported in part by the Natural Sciences and Engineering Research Council (NSERC) of Canada, the Advanced Sensor Networks SARChI Chair funding from the National Research Foundation of South Africa and the National Research Foundation (NRF) of South Africa and the DTI THRIP Program. 


\section{REFERENCES}

[1] J. Mitola and G. Q. M. Jr, "Cognitive radio: Making software radios more personal," IEEE Personal Communications, vol. 6, pp. 13-18, Aug 1999.

[2] J. Mitola, Cognitive Radio: An Integrated Agent Architecture for Software Defined Radios. PhD thesis, KTH,Sweden, 2000.

[3] S. Haykin, "Cognitive radio: Brain-empowered wireless communications," IEEE Journal on Selected Areas in Communications, vol. 23, Feb 2005.

[4] M. R. Garey and D. S. Johnson, Computers and Intractability: A Guide to the Theory of NP-Completeness. W. H. Freeman \& Co., 1979.

[5] P. Cheng, Z. Zhang, H. Huang, and P. Qiu, "A distributed algorithm for optimal resource allocation in cognitive OFDMA systems," IEEE International Conference on Communications, May 2008.

[6] S. M. Almalfouh and G. L. Stuber, "Interference-aware radio resource allocation in OFDMA-based cognitive radio networks," IEEE Transactions on Vehicular Technology, vol. 60, pp. 1699-1713, May 2011.

[7] S. Wang, Z. H. Zhou, M. Ge, and C. Wang, "Resource allocation for heterogeneous cognitive radio networks with imperfect spectrum sensing," IEEE Journal of Selected Areas in Communication, vol. 31, pp. 464-475, March 2013.

[8] S. Boyd and C. Vandenberghe, Convex Programming. Cambridge University Press, 2004.

[9] M. L. Fisher, "The Lagrangian relaxation relaxation method for solving integer programming problems," Management Sciences, vol. 27, no. 1, pp. 1-18, 1981.

[10] M. L. Fisher, "An application oriented guide to Lagrangian relaxation," Interfaces, vol. 15, no. 2, pp. 10-21, 1985.

[11] Y. Rahulamathavan, K. Cumanan, L. Musavian, and S. Lambotharan, "Optimal subcarrier and bit allocation techniques for cognitive radio networks using integer linear programming," Statistical Signal Processing (SSP), IEEE Workshop, Sept 2009.

[12] P. Mitran, L. Le, C. Rosenberg, and A. Girard, "Resource allocation for downlink spectrum sharing in cognitive radio networks," IEEE Vehicular Technology Conference, Sept 2008.

[13] Y. Shi and Y. T. Hou, "A distributed optimization algorithm for multi-hop cognitive radio networks," IEEE INFOCOM, April 2008.

[14] M. Ma and D. H. K. Tsang, "Joint spectrum sharing and fair routing in cognitive radio networks," IEEE Consumer Communications and Networking Conference (CCNC), Jan 2008.

[15] M. Ma and D. H. K. Tsang, "Joint design of spectrum sharing and routing with channel heterogeneity in cognitive radio networks," ELSEVIER Physical Communication Journal, vol. 2, pp. 127-137, March-June 2009.

[16] T. Shu and M. Krunz, "Coordinated channel access in cognitive radio networks: A multi-level spectrum opportunity perspective," IEEE INFOCOM, April 2009

[17] B. S. Awoyemi, B. T. Maharaj, and A. S. Alfa, "Resource allocation for heterogeneous cognitive radio networks," IEEE WCNC, March 2015.

[18] Y. Rahulamathavan, K. Cumanan, and S. Lambotharan, "Optimal resource allocation techniques for MIMO-OFDMA based cognitive radio networks using integer linear programming," Signal Processing Advances in Wireless Communications(SPAWC) IEEE International Workshop, June 2010.

[19] W. Guo and X. Huang, "Maximizing throughput for overlaid cognitive radio networks," IEEE Military Communications Conference (MILCOM), Oct 2009.

[20] C. F. Shih, W. Liao, and H. L. Chao, "Joint routing and spectrum allocation for multi-hop cognitive radio networks with route robustness consideration," IEEE Transactions on Wireless Communications, vol. 10, pp. 29402949, Sept 2011.

[21] W. Wang, T. Lv, Z. Ren, L.Gao, and W. Liu, "A novel spectrum sharing algorithm based on the throughput in cognitive radio networks," Wireless Communications, Networking and Mobile Computing (WiCOM), Sept 2009.

[22] A. T. Hoang and Y. C. Liang, "Downlink channel assignment and power control for cognitive radio networks," IEEE Transactions on Wireless Communications, vol. 7, pp. 3106-3117, Aug 2008.

[23] A. T. Hoang and Y. C. Liang, "Maximizing spectrum utilization of cognitive radio networks using channel allocation and power control," IEEE Vehicular Technology Conference (VTC), Sept 2006.

[24] Y. Rahulamathavan, K. Cumanan, R. Krishna, and S. Lambotharan, "Adaptive subcarrier and bit allocation techniques for MIMO-OFDMA based uplink cognitive radio networks," UK-India International Workshop on Cognitive Wireless Systems (UKIWCWS), Dec 2009.

[25] C. An, R. Xie, H. Ji, and Y. Li, "Pricing and power control for energyefficient radio resource management in cognitive femtocell networks," International Journal of Communication Systems, vol. 28, pp. 743-761, Mar. 2015.

[26] P. Mitran, L. Le, and C. Rosenberg, "Queue-aware resource allocation for downlink OFDMA cognitive radio networks," IEEE Transactions on Wireless Communications, vol. 9, pp. 3100-3111, Oct. 2010.

[27] M. Waheed and A. Cai, "Evolutionary algorithms for radio resource management in cognitive radio network," IEEE Performance Computing and Communications Conference, Dec 2009.

[28] D. T. Ngo, C. Tellambura, and H. H. Nguyen, "Resource allocation for OFDMA-based cognitive radio multicast networks with primary user activity consideration," IEEE Transactions on Vehicular Technology, vol. 59, pp. 1668 - 1679, May 2010.

[29] H. B. Salameh, "Rate-maximization channel assignment scheme for cognitive radio networks," IEEE Global Telecommunication Conference (GLOBECOM), Dec 2010.

[30] Y. Zhang and C. Leung, "Resource allocation in an OFDM-based cognitive radio system," IEEE Transactions on Communications, vol. 57, pp. 1928 - 1931, July 2009.

[31] R. Xie, F. R. Yu, and H. Ji, "Joint power allocation and beamforming with users selection for cognitive radio networks via discrete stochastic optimization," IEEE Global Telecommunication Conference (GLOBECOM), Dec 2011.

[32] W. J. Shin, K. Y. Park, D. I. Kim, and J. W. Kwon, "Large-scale joint rate and power allocation algorithm combined with admission control in cognitive radio networks," Journal of Communication and Networks, vol. 11, pp. 157 - 165, April 2009.

[33] P. Li, S. Guo, W. Zhuang, and B. He, "On efficient resource allocation for cognitive and cooperative communications," IEEE Journal on Selected Areas in Communications, vol. 32, pp. 264-273, February 2014.

[34] H. Xu and B. Li, "Resource allocation with flexible channel cooperation in cognitive radio networks," IEEE INFOCOM, Mar 2010.

[35] S. M. Almalfouh and G. L. Stuber, "Uplink resource allocation in cognitive radio networks with imperfect spectrum sensing," IEEE Vehicular Technology Conference, Sept 2010.

[36] Y. Zhang and C. Leung, "A distributed algorithm for resource allocation in OFDM cognitive radio systems," IEEE Transactions on Vehicular Technology, vol. 60, pp. 546-554, Feb 2011.

[37] H. Yu, W. Tang, and S. Li, "Joint optimal sensing and power allocation for cooperative relay in cognitive radio networks," IEEE International Conference on Communications (ICC), June 2012.

[38] C. Zhao and K. Kwak, "Joint sensing time and power allocation in cooperatively cognitive networks," IEEE Communication Letters on Communications, vol. 14, Feb 2012.

[39] J. Jia, J. Zhang, and Q. Zhang, "Cooperative relay for cognitive radio networks," IEEE INFOCOM, April 2009.

[40] W. Yao, Y. Wang, and T. Wang, "Joint optimization for downlink resource allocation in cognitive radio cellular networks," IEEE Consumer Coтmunications and Networking Conference (CCNC), Jan 2011.

[41] D. T. Ngo and T. L. Ngoc, "Distributed resource allocation for cognitive radio networks with spectrum-sharing constraints," IEEE Transactions on Vehicular Technology, vol. 60, Sept 2011.

[42] P. Lin and T. Lin, "Optimal dynamic spectrum access in multi-channel multi-user congitive radio networks," IEEE International Symposium on Personal, Indoor and Mobile Radio Communications, 2010.

[43] W. Wang, K. G. Shin, and W. Wang, "Joint spectrum allocation and power control for multihop cognitive radio networks," IEEE Transactions on Mobile Computing, vol. 10, July 2011.

[44] D. Xu, Z. Feng, Y. Li, and P. Zhang, "Fair channel allocation and power control for uplink and downlink cognitive radio networks," IEEE Global Telecommunication Conference (GLOBECOM) Workshops, Dec 2011.

[45] Z. Golcezaei-Khuzani and M. Ardebilipour, "Resource allocation for cognitive relay networks," 17th Telecommunications Forum TELFOR, Nov 2009.

[46] S. Wang, M. Ge, and C. Wang, "Efficient resource allocation for cognitive radio networks with cooperative relays," IEEE Journal on Selected Areas in Communications, vol. 31, pp. 264-273, November 2013.

[47] M. Shaat, T. Ngatched, and O. Dobre, "Resource allocation in OFDMbased cognitive two-way multiple relay network," IEEE Globecom, 2014.

[48] R. Fan, H. Jiang, Q. Guo, and Z. Zhang, "Joint optimal cooperative sensing and resource allocation in multichannel cognitive radio networks," IEEE Transactions on Vehicular Technology, vol. 60, Feb 2011.

[49] R. Xie, F. R. Yu, and H. Ji, "Dynamic resource allocation for heterogeneous services in cognitive radio networks with imperfect channel sensing," IEEE Transactions on Vehicular Technology, vol. 61, pp. 770-780, Feb 2012.

[50] R. Xie, H. Ji, P. Si, and Y. Li, "Dynamic channel and power allocation in cognitive radio networks supporting heterogeneous services," IEEE Global Telecommunication Conference (GLOBECOM), Dec 2010.

[51] M. V. Nguyen, J. Yoon, J. Lee, and H. S. Lee, "Scheduling in cellular cognitive networks," IEEE Wireless Communications and Network Conference $(W C N C)$, Mar 2011. 
[52] J. Xiang, Y. Zhang, T. Skeie, and L. Xie, "Downlink spectrum sharing for cognitive radio femtocell networks," IEEE Systems Journal, vol. 4, pp. 524-534, Dec 2010.

[53] F. Huang, S. Wang, and S. Du, "Resource allocation in OFDM-based multi-cell cognitive radio systems," IEEE Wireless and Optical Communications Conference, April 2011.

[54] S. M. Almalfouh and G. L. Stuber, "Inteference-aware power allocation in cognitive radio networks with imperfect spectrum sensing," IEEE International Conference on Communications (ICC), May 2010.

[55] K. W. Choi, E. Hossain, and D. I. Kim, "Downlink subchannel and power allocation in multi-cell OFDMA cognitive radio networks," IEEE Transactions on Wireless Communications, vol. 10, pp. 2259 - 2271, July 2011.

[56] D. Hu and S. Mao, "Co-channel and adjacent channel interference mitigation in cognitive radio networks," Military Communications Conference (MILCOM), Nov 2011

[57] S. Wang, F. Huang, and C. Wang, "Adaptive proportional fairness resource allocation for OFDM-based congitive radio networks," Wireless Networks, vol. 19, pp. 273 - 284, April 2013.

[58] K. L. Du, M. N. S. Swamy, and Q. Ni, "A dynamic spectrum access scheme for cognitive radio networks," IEEE Canadian Conference on Electrical and Computer Engineering (CCECE), May 2009.

[59] L. Li, C. Xu, P. Fan, and J. He, "Resource allocation in orthogonal frequency division multiple access-based cognitive radio systems with minimum rate constraints," International Journal of Communication Systems, August 2012.

[60] K. Cumanan, R. Krishna, L. Musavian, and S. Lambotharan, "Joint beamforming and user maximization techniques for cognitive radio networks based on branch and bound method," IEEE Transactions on Wireless Communications, vol. 9, pp. 3082-3092, Oct 2010.

[61] Y. Shi, Y. Hou, and H. Zhou, "Per node based optimal power control for multi-hop cognitive radio networks," IEEE Transactions on Wireless Communications, vol. 8, pp. 5290-5299, Oct 2009.

[62] P. K. Tseng, W. H. Chung, H. Chen, and C. S. Wu, "Distributed energyefficient cross-layer design for cognitive radio networks," Personal Indoor and Mobile Radio Communications (PIMRC), IEEE International Symposium, Sept 2012.

[63] Y. T. Hou, Y. Shi, and H. D. Sherali, "Spectrum sharing for multi-hop networking with cognitive radios," IEEE Journal on Selected Areas in Communication, vol. 26, pp. 146-155, Jan 2008.

[64] F. Hou and J. Huang, "Dynamic channel selection in cognitive radio network with channel heterogeneity," IEEE Global Telecommunication Conference (GLOBECOM), Dec 2010.

[65] J. C. Liang and J. C. Chen, "Resource allocation in cognitive radio relay networks," IEEE Journal on Selected Areas in Communication, vol. 31, pp. 476 - 488, March 2013.

[66] Y. Tachwali, B. F. Lo, I. F. Akyildiz, and R. Agusti, "Multiuser resource allocation optimization using bandwidth-power product in cognitive radio networks," IEEE Journal on Selected Areas in Communication, vol. 31, pp. $451-463$, March 2013.

[67] L. Zhang, Y. C. Liang, and Y. Xin, "Joint admission control and power allocation for cognitive radio networks," IEEE International Conference on Acoustics, Speech and Signal Processing (ICASSP), April 2007.

[68] Q. Lu, T. Peng, W. Wang, and W. Wang, "Optimal subcarrier and power allocation under interference temperature constraints," IEEE Wireless Communications and Networks Conference (WCNC), April 2009.

[69] X. Chen and C. Yuen, "Efficient resource allocation in a rateless-coded MU-MIMO cognitive radio network with QoS provisioning and limited feedback," IEEE Transactions on Vehicular Technology, vol. 62, Jan 2013.

[70] Z. Guan, L. Ding, T. Melodia, and D. Yuan, "On the effect of cooperative relaying on the performance of video streaming applications in cognitive radio networks," IEEE International Conference on Communications (ICC), June 2011.

[71] A. A. El-Sherif and A. Mohamed, "Joint routing and resource allocation for delay minimization in cognitive radio based mesh networks," IEEE Transactions on Wireless, vol. 13, pp. 186-197, January 2014.

[72] K.M.Thilina, M. Moghadari, and E. Hossain, "Generalized spectral footprint minimization for OFDMA-based cognitive radio networks," IEEE ICC, 2014

[73] Y. Chen, Q. Lei, and X. Yuan, "Resource allocation based on dynamic hybrid overlay/underlay for heterogeneous services of cognitive radio networks," IEEE Globecom, pp. 1647-1664, 2014.

[74] Q. Lei and Y. Chen, "Resource allocation based on hybrid sharing mode for heterogeneous services of cognitive radio ofdm systems," KSII Transactions on Internet and Information Systems, vol. 9, no. 1, pp. 149-168, 2015.

[75] H. Zhang, C. Jiang, N. C. Beaulieu, X. Chu, X. Wang, and T. Q. S. Quek, "Resource allocation for cognitive small cell networks: A cooperative bar- gaining game theoretic approach," IEEE Transactions on Wireless Communications, vol. 14, pp. 3481-3493, Jun. 2015.

[76] H. Zhang, C. Jiang, X. Mao, and H. Chen, "Interference-limited resource optimization in cognitive femtocells with fairness and imperfect spectrum sensing," IEEE Transactions on Vehicular Technology, vol. PP, pp. 1-11, Feb. 2015.

[77] J. Chen, J. Chu, and K. Feng, "Energy-efficient spectrum selection and resource allocation in downlink cognitive femtocell networks," IEEE Vehicular Technology Conference (VTC Spring), May 2015

[78] D. Ng, E. S. Lo, and R. Schober, "Interference-limited resource optimization in cognitive femtocells with fairness and imperfect spectrum sensing," IEEE Transactions on Wireless Communications, vol. 11, pp. 3618-3631, Oct. 2012

[79] L. Li, C. Xu, and M. Tao, "Resource allocation in open access ofdma femtocell networks," IEEE Wireless Communications Letters, vol. 1, pp. 625628, Sep. 2012.

[80] G. Zhang, X. Ao, P. Yang, and M. Li, "Power management in adjacent cognitive femtocells with distance-dependent interference in full coverage area," EURASIP Journal on Wireless Communications and Networking, vol. 2016, pp. 1-10, Jan. 2016.

[81] J. P. M. Torregoza, R. Enkhbat, and W. Hwang, "Joint power control, base station assignment, and channel assignment in cognitive femtocell networks," EURASIP Journal on Wireless Communications and Networking, vol. 2010, pp. 1-14, May 2010.

[82] P. Phunchongharn, E. Hossain, K. W. Choi, and S. Camorlinga, "Robust transmission scheduling and power control for spectrum sharing in spatial reuse TDMA wireless networks," IEEE Globecom, Dec 2010.

[83] P. Phunchongharn, E. Hossain, L. Le, and S. Camorlinga, "Robust scheduling and power control for vertical spectrum in STDMA wireless networks," IEEE Transactions on Wireless Communications, vol. 11, pp. 1850-1860, Mar 2012.

[84] P. C. Gilmore and R. E. Gomory, "A linear programming approach to the cutting-stock problem,” Operations Research, vol. 9, pp. 849-854, 1961.

[85] P. C. Gilmore and R. E. Gomory, "A linear programming approach to the cutting-stock problem - Part II," Operations Research, vol. 119, pp. 863 $888,1963$.

[86] N. Karmarkar, "A new polynomial-time algorithm for linear programming," Combinatorica, vol. 4, no. 4, pp. 373-395, 1984.

[87] S. Mehrotra, "On the implementation of a primal-dual interior point method," SIAM Journal on Optimization, vol. 2, no. 4, pp. 575-601, 1992.

[88] G. L. Nemhauser and L. A. Wolsey, Integer and Combinatorial Optimization. Wiley-Interscience, 1988

[89] A. Caprara and M. Fischetti, "Branch-and-cut algorithms," Annotated Bibiliographies in Combinatorial Optimization, pp. 45-63, 1997.

[90] A. Geoffrion, "Lagrangean relaxation for integer programming," Math. Programming Stud., vol. 2, pp. 82-114, 1974.

[91] A. Abadpour, A. S. Alfa, and A. K. Soong, "Closed form solution for maximizing the sum capacity of reverse link CDMA system with rate constraints," IEEE Transactions on Wireless Communications, vol. 7, pp. 1179-1183, Apr 2008 .

[92] A. Ibrahim and A. S. Alfa, "Radio resource allocation for multicast transmissions over high altitude platforms," IEEE Globecom Workshop, pp. 281-287, 2013.

[93] J. F. Benders, "Partitioning procedures for solving mixed variables programming problems," Numer. math., vol. 4, pp. 238-252, 1962.

[94] A. M. Geoffrion, "Generalized benders decomposition," J. Optimization Theory Applic., vol. 10, no. 4, pp. 237-260, 1972.

[95] M. J. Bagajewwicz and V. Manousiouthakis, "On the generalized benders decomposition," Computers Chem. Engng, vol. 15, no. 10, pp. 691-700, 1991.

[96] F. Eisenbrand, Fast integer programming in fixed dimension. Springer: Lecture Notes in Comput. Sci. 2832, 2003.

[97] H. W. Lenstra, "Integer programming with a fixed number of variables," Mathematics of Operations Research, vol. 8, no. 4, pp. 538-548, 1983. 


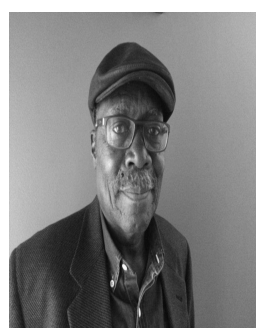

Attahiru S. Alfa is a professor of telecommunication systems at the University of Manitoba, Department of Electrical and Computer Engineering and also a SARChI Chair professor at the University of Pretoria, Department of Electrical, Electronic and Computer Engineering. His research covers, but not limited to, the following areas: queueing theory and applications, optimization, performance analysis and resource allocation in telecommunication systems, modeling of communication networks, analysis of cognitive radio networks, modeling and analysis of wireless sensor networks, developing efficient decoding algorithms for LDPC codes, channel modeling, traffic estimation for the Internet, and cross layer analysis. Dr. Alfa also works in the application of queueing theory to other areas such as transportation systems, manufacturing systems and healthcare systems. He has authored two books, "Queueing Theory for Telecommunications: Discrete Time Modelling of a Single Node System", published by Springer in 2010, and "Applied Discrete-Time Queue" published in 2015 also by Springer.

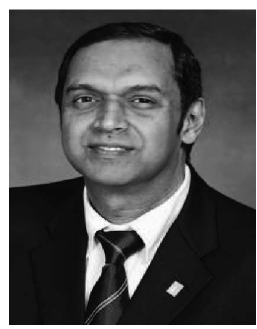

Sunil Maharaj received his $\mathrm{PhD}$ in Wireless Communications from the University of Pretoria. Professor Maharaj currently holds the position of Sentech Chair in Broadband Wireless Multimedia Communications in the Department of Electrical, Electronic and Computer Engineering at the University of Pretoria. His research interests are in OFDM-MIMO systems, massive MIMO and cognitive radio resource allocation.

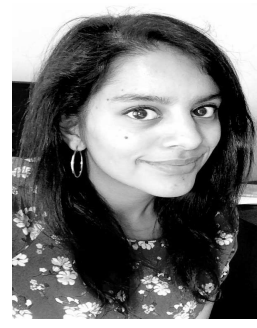

Shruti Lall received her BEng in computer engineering from the University of Pretoria in 2013. She completed her honours in electronic engineering also from the University of Pretoria in 2014. She has subsequently completed her MEng in the Broadband Wireless Multimedia Communications group in 2015. Her research interests are in wireless network security, optimisation and wireless mesh networks.

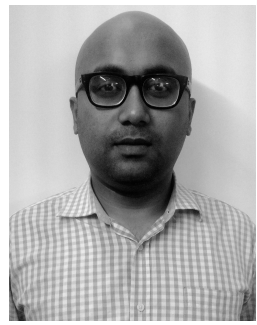

Sougata Pal received his M.Sc in Mobile Communication Engineering in 2009 from "Institute Eurecom" which is a top French Research and Development Institute on Communication Engineering. Institute Eurecom comes under the prestigious French Telecommunication Engineering School, "Telecom ParisTech". He received his Bachelors in Electronics and Telecommunication Engineering in 2004 from Haldia Institute of Technology, India. Since August, 2014 Sougata is working as a Patent Engineer in the field of Intellectual Property Rights (IPR) dealing with Innovations in the fields of Internet of Things (IOT), Telecommunications, Software, Interactive Voice Response (IVR) Systems etc. Prior to that he has worked in Telecommunication Industry, Academic Research and Education Management for 7+ years in different countries (India/France/Spain/South Africa/Singapore). During his tenure working as an Academic Researcher he has worked in the fields of Sensor Networks, IP Multimedia Subsystems etc. 